\title{
Mobilisierung des Rechts: Organisierte Interessen und Verfassungsbeschwerden vor dem Bundesverfassungsgericht
}

\author{
Stefan Thierse
}

Online publiziert: 15 . April 2020

(C) Der/die Autor(en) 2020

Zusammenfassung Verfassungsbeschwerden werden nicht nur von Einzelpersonen erhoben, sondern auch von Unternehmen, Verbänden oder zivilgesellschaftlichen Interessengruppen. Gleichwohl sind die politischen Implikationen des Verfahrens von der Politikwissenschaft bisher kaum gewürdigt worden. Dies ist umso bemerkenswerter, als sogar die Rechtswissenschaft der Verfassungsbeschwerde eine Mobilisierungs- und Partizipationsfunktion zuerkennt. Vor diesem Hintergrund wirft der vorliegende Beitrag die folgende Frage auf: Unter welchen Bedingungen erreichen organisierte Interessen mithilfe einer Verfassungsbeschwerde eine breite Mobilisierung für ihre Anliegen? Eine Fuzzy-set-Analyse von 35 Verfassungsbeschwerden vor dem Bundesverfassungsgericht identifiziert zwei kausale Konfigurationen für das Phänomen verfassungsrechtlicher Mobilisierungsstrategien. Bei allen Unterschieden teilen diese Verfassungsbeschwerden den Umstand, dass sie von Mitgliederorganisationen erhoben werden, die in Karlsruhe Gesetze und Regierungsakte anfechten.

Schlüsselwörter Politische Gelegenheitsstruktur · Interessengruppen · Rechtsmobilisierung · Massenverfassungsbeschwerde $\cdot$ Fuzzy-set-QCA

Eine frühere Version dieses Artikels wurde auf der Jahrestagung des Arbeitskreises Organisierte Interessen der DVPW am 28./29. November 2018 an der Universität Bielefeld präsentiert.

Zusatzmaterial online Zusätzliche Informationen sind in der Onlineversion dieses Artikels (https:// doi.org/10.1007/s11615-020-00235-5) enthalten.

S. Thierse $(\bowtie)$

Institut für Europastudien, Universität Bremen, Bibliothekstraße 1, 28359 Bremen, Deutschland

E-Mail: thierse@uni-bremen.de 


\title{
Legal Mobilization: Organized Interests and Constitutional Complaints Before the Federal Constitutional Court
}

\begin{abstract}
Constitutional complaints are not only lodged by private individuals, they are also brought by firms, associations, and public interest groups. However, the political implications of the procedure have gone largely unnoticed by political science. This is all the more remarkable given that even the mobilizing and participatory function of the constitutional complaint is acknowledged by legal theory. Against this backdrop, the present article raises the following question: under what conditions do organized interests achieve broad mobilization for their causes by means of a constitutional complaint? A fuzzy set analysis of 35 constitutional complaints before the Federal Constitutional Court identifies two causal configurations for the phenomenon of constitutional mobilizing strategies. Despite all the differences, these constitutional complaints share the condition that they are brought by membership organizations that challenge statutes and acts of government in Karlsruhe.
\end{abstract}

Keywords Political opportunity structure · Interest groups · Legal mobilization · Mass constitutional complaint · Fuzzy set qualitative comparative analysis

\section{Einleitung}

„Verfassungsklage per Menschenkette“, „125.000 Mal „Nein“ zu CETA“, „125.000 CETA-Gegner unterstützen Verfassungsklage"; so oder ähnlich titelten deutsche Tageszeitungen und Nachrichtenportale im August 2016, als Vertreter der drei Nichtregierungsorganisationen (NGOs) Campact, foodwatch und Mehr Demokratie e. V. Verfassungsbeschwerde gegen das Comprehensive Economic and Trade Agreement (CETA) beim Bundesverfassungsgericht (BVerfG) einreichten. Die „Bürgerklage“ ist eine von insgesamt vier Verfassungsbeschwerden gegen das Handels- und Investitionsabkommen zwischen der Europäischen Union (EU) und Kanada. Eine ähnlich breite Mobilisierung der Zivilgesellschaft gab es zuvor erstmals gegen das Umsetzungsgesetz zur EU-Richtlinie über die Vorratsdatenspeicherung (Thierse und Badanjak 2020).

Die Nutzung von Verfassungsbeschwerden zur „Mobilisierung des Rechts“ (Blankenburg 1995) verweist auf ein empirisches Rätsel: Rein rechtlich ist es unerheblich, ob eine Verfassungsbeschwerde von einem oder 100.000 Klägern eingereicht wird. Das Urteil eines Verfassungsgerichts sollte nach der Prüfung des Falls unabhängig von der Anzahl der Beschwerdeführer gleich lauten. Warum setzen manche Akteure die Verfassungsbeschwerde zur Mobilisierung für politische Anliegen ein? Und welche Faktoren tragen zu einer breiten politischen Mobilisierung bei? Diese Fragen haben bislang erstaunlich wenig Resonanz in der politikwissenschaftlichen Forschung gefunden. In dieses Bild passt, dass Verfassungsbeschwerden trotz ihrer nicht nur in der Bundesrepublik quantitativ überragenden Bedeutung am Arbeitsanfall von Verfassungsgerichten (Hönnige 2010, S. 352; Visser 2015, S. 154) bislang kaum empi- 
risch aufgearbeitet sind. ${ }^{1}$ Der vorliegende Beitrag adressiert diese Forschungslücke, indem er den Zugang zur verfassungsgerichtlichen Überprüfung staatlichen Handelns als Teil der politischen Gelegenheitsstruktur konzeptualisiert. Inspiriert durch die Literatur zur Rechtsmobilisierung (,legal mobilization“) (Blankenburg 1995; Epp 2008a, b; McCann 2008) knüpft der Artikel an die Idee an, dass das Recht bzw. der Rückgriff auf Rechtsmittel nicht mechanistisch politischen und sozialen Wandel bewirkt, sondern bestenfalls eine Ressource darstellt, die sich in politischen Einfluss ummünzen lässt. Das Erkenntnisinteresse dieses Beitrags liegt folglich auf einer besonderen, zahlenmäßig marginalen Gruppe von Verfassungsbeschwerden: solchen, die zur Durchsetzung politischer Kurskorrekturen von organisierten Interessen der Zivilgesellschaft initiiert und organisiert werden und auf die Herstellung kritischer Öffentlichkeit abzielen.

Der Beitrag verfährt wie folgt: Im nächsten Abschnitt werden grundlegende Voraussetzungen und mögliche Wirkungen von Verfassungsbeschwerden unter dem Gesichtspunkt der politischen Gelegenheitsstruktur diskutiert. Unter Rückgriff auf eine von Schreier (2011) vorgeschlagene Typologie werden sog. Massenverfassungsbeschwerden von einem gewöhnlichen Rechtsbehelf abgegrenzt. Anschließend werden in Abschn. „Theoretischer Rahmen und Hypothesen“ unter Rückgriff auf Erkenntnisse der Interessengruppenforschung Überlegungen angestellt, welche Akteure den größten Anreiz zur Nutzung von Verfassungsbeschwerden als Mobilisierungsinstrument haben. Abschn. „Verfassungsbeschwerden von Interessengruppen: Daten und Methode" stellt die Datengrundlage und Methode vor und liefert eine empirische Analyse von 35 Verfassungsbeschwerden im Zeitraum 2000 bis 2017. In Abschn. „Analyse und Diskussion“ werden die Ergebnisse einer Fuzzy-set-Analyse präsentiert. Der Abschn. ,Schlussbetrachtung und Ausblick“ diskutiert die Ergebnisse, ordnet diese in den Stand der Forschung ein und skizziert weiterführende Forschungsfragen und mögliche Anknüpfungspunkte.

\section{Verfassungsbeschwerden als Teil der politischen Gelegenheitsstruktur und als Instrument der Rechtsmobilisierung}

Verfassungsbeschwerden bieten neben abstrakter und konkreter Normenkontrolle einen dritten Verfahrensweg für die verfassungsgerichtliche Überprüfung von Akten staatlicher Gewalt (Stone Sweet 2000, S. 44-45). Aus einer strikt rechtswissenschaftlichen Sicht ist die Verfassungsbeschwerde ein außerordentlicher Grundrechtsbehelf. „Jedermann“, der sich durch einen Akt öffentlicher Gewalt persönlich, gegenwärtig und unmittelbar in seinen Grundrechten oder grundrechtsgleichen Rechten verletzt sieht, steht prinzipiell der Weg der Verfassungsbeschwerde offen. ${ }^{2}$ Das Erfordernis, mit der Erhebung einer Verfassungsbeschwerde die persönliche, gegenwärtige und unmittelbare Betroffenheit nachzuweisen, ist die zentrale Voraussetzung, um über-

\footnotetext{
1 Die wenigen Arbeiten, die sich in vergleichender Perspektive mit Verfassungsbeschwerden befassen, stammen aus der Rechtswissenschaft (Brunner 2002; Dannemann 1994; Visser 2015).

2 Auch juristische Personen fallen grundsätzlich unter ,,jedermann“, sind also beschwerdefähig, sofern sie Träger von Grundrechten sind (Visser 2015).
} 
haupt Zugang zum Verfassungsgericht zu erhalten und verweist auf die Tatsache, dass das Gerichtssystem im Gegensatz zu anderen Subsystemen auf individuelles Handeln ausgerichtet ist (Zemans 1983, S. 692). Folglich ist die Verfassungsbeschwerde auch nicht dazu gedacht, wie bei einer Popularklage Rechte Dritter geltend zu machen. Ebenso soll die Voraussetzung, den Schutz durch die Verfassung geschützter Grundrechte zunächst vor Fachgerichten zu erwirken, dem „Kurzschluss“ des Instanzenwegs vorbeugen. Schließlich - damit zusammenhängend - befasst sich das Verfassungsgericht ausschließlich mit verfassungsrechtlichen Fragen, was aus Sicht der Kläger eine weitere Einschränkung impliziert (Dannemann 1994, S. 142).

Diese formal voraussetzungsvollen Zugangsbedingungen scheinen potenzielle Beschwerdeführer jedoch nur bedingt abzuschrecken. Jährlich gehen fast $6000 \mathrm{Ver}-$ fassungsbeschwerden in Karlsruhe ein, ihr Anteil an allen Verfahrensarten liegt im langfristigen Durchschnitt (1951-2017) bei nahezu 97 Prozent. ${ }^{3}$ Selbst Vertreter der Rechtswissenschaft anerkennen, dass Verfassungsbeschwerden neben ihrer (verfassungs-)rechtlichen Funktion des Grundrechtsschutzes sowie der Rechtsfortbildung eine dritte Funktion erfüllen: die der ,Mobilisierung der Bürger für die Durchsetzung von Verfassungsrecht" und der wirksamen Verteidigung ihrer Grundrechte gegenüber dem Staat (Gusy 2015, S. 344-345; Zuck 2013, S. 35). Blankenburg (1998, S. 217) charakterisiert Verfassungsbeschwerden daher recht unverblümt als „Nebenbühne der Politik und Klagemauer von Bürgern“.

Verfassungsbeschwerden sind Teil der politischen Gelegenheitsstruktur: Sie stellen Handlungsoptionen dar, deren Nutzung indes mit Chancen, Risiken, Unwägbarkeiten und Zielkonflikten einhergehen (Koopmans 1999). Aus Sicht der Beschwerdeführer ist das sicherlich wichtigste Motiv, eine Entscheidung zu erwirken, die einen als verfassungswidrig gehaltenen Eingriff in die Grundrechte heilt. Insbesondere Verfassungsbeschwerden, die sich unmittelbar gegen Gesetze richten (Rechtssatzverfassungsbeschwerden), können politische Entscheidungen revidieren oder konditionieren, sofern das BVerfG ein Gesetz für verfassungswidrig erklärt und als nichtig verwirft. Das Kontrollpotenzial ist aufgrund der Verwerfungskompetenz des Verfassungsgerichts als größer einzuschätzen als bei anderen Handlungsoptionen, die Bürgern oder Interessengruppen zu Gebote stehen, denn die Anrufung des Verfassungsgerichts schafft unter Umständen schneller Abhilfe gegen invasive Entscheidungen als die Aussicht auf Abwahl der Regierung bei den nächsten Wahlen. Dies gilt insbesondere dann, wenn die Verfassungsbeschwerde mit einem Antrag auf einstweiligen Rechtsschutz verbunden wird. Je länger jedoch der Zeitraum zwischen Klage und Urteilsspruch ausfällt, umso unrealistischer ist die Aussicht auf eine rasche Korrektur politischer Entscheidungen (Brandenburg 2014; Köhler 2012, S. 210). ${ }^{4}$ Stone Sweet (2000, S. 199) spitzt die potenzielle Wirkung von Verfas-

\footnotetext{
3 Eigene Berechnungen auf Basis der vom BVerfG veröffentlichen Übersicht über die Verfahrenseingänge. https://www.bundesverfassungsgericht.de/DE/Verfahren/Jahresstatistiken/2017/gb2017/A-I-4.pdf?_ blob=publicationFile \&v=2 (zuletzt abgerufen am 18.10.2019).

${ }^{4}$ In immerhin mehr als einem Drittel aller Verfassungsbeschwerden vergehen bis zu einer Entscheidung zwei Jahre oder mehr. https://www.bundesverfassungsgericht.de/DE/Verfahren/Jahresstatistiken/2017/ gb2017/A-IV-3.pdf?_blob=publicationFile \&v=2.
} 
sungsbeschwerden als Ergänzung der politischen Einflussnahme folgendermaßen $\mathrm{zu}$ :

Private individuals, companies, and interest groups who want to provoke changes in statutes, and to build constraints on the exercise of legislative power in the future, have an interest in bringing litigation that raises constitutional objections. [...] The result is that judicial processes have become sites of policymaking that supplement, and at times rival, the legislature. In systems in which the individual complaint procedure exists, this outcome is reinforced, since litigants can appeal judicial decisions (denying standing or their constitutional claims) and statutes (under certain conditions) directly to the constitutional court. [eigene Herv.]

Der Anreiz, mittels einer Verfassungsbeschwerde ein Gesetz zu Fall zu bringen, dürfte insbesondere für zivilgesellschaftliche Interessengruppen noch größer sein als für Unternehmen und Wirtschaftsverbände. Rechtsstreitigkeiten - die Verfassungsbeschwerden zumindest dem Verfahren nach sind - lassen sich als eine alternative Strategie der Interessenvermittlung und Konfliktregelung neben dem direkten Kontakt zu Entscheidungsträgern (Lobbying) und Protest interpretieren (Hilson 2002, S. 239; McCown 2009, S. 91). Der Rekurs auf das Recht und Rechtsmittel gilt in der Interessengruppenforschung als Merkmal von Gruppen, die im Zugang zu repräsentativen Institutionen oder zur Verwaltung strukturell unterlegen sind und ihre materiell-inhaltlichen politischen Zielsetzungen anders kaum zur Geltung bringen können (Cortner 1968, S. 287). Rechtsstreitigkeiten strukturell schwacher Gruppen dienen dazu, durch eine Ausweitung oder Reinterpretation von Rechten eine Veränderung des Status quo herbeizuführen - wohingegen Rechtsstreitigkeiten strukturell starker Gruppen der Verteidigung des Status quo dienen (Cortner 1968, S. 288; Gawron und Schäfer 1976, S. 240).

In der Literatur zu sozialen Bewegungen hat sich ein eigenständiger Forschungsstrang etabliert, der das Potenzial von Rechtsstreitigkeiten zur Artikulation und Durchsetzung politischer Reformanliegen in vielen Politikfeldern ergründet (Andersen 2005; Burstein 1991; Handler 1978; Epp 2008b). Rechtsmobilisierung lässt sich allgemein begreifen als Berufung auf Rechtsansprüche zur Regulierung des Verhaltens anderer Akteure (Zemans 1983, S. 700). Die Bewegungsforschung fokussiert vor allem auf Rechtsmobilisierung kollektiver Akteure, auf Rechtsstreitigkeiten vor Höchst- und Verfassungsgerichten und eine spezifische Form der Rechtsmobilisierung: strategische Prozessführung. Die vielfältigen Definitionsversuche (Graser und Helmrich 2019) lassen sich dahingehend bündeln, dass es sich bei strategischer Prozessführung der Form nach um sozialen Aktivismus mit den Mitteln des Rechts bzw. um nicht verfasste politische Partizipation handelt, die von sog. Klagekollektiven (Hahn 2019, S. 19) aus Beschwerdeführern, Interessengruppen, Anwälten, finanziellen und ideellen Unterstützern und Sympathisanten initiiert und getragen wird. Inhaltlich zielt strategische Prozessführung jenseits des etwaigen kurzfristigen Erfolgs vor Gericht auf die langfristige Veränderung politischer und gesellschaftlicher (Macht-)Verhältnisse. Geradezu konstitutiver Bestandteil strategischer Prozessführung ist eine professionelle Öffentlichkeitsarbeit, mit der breite Aufmerksamkeit 
und ein Problembewusstsein für eine dem Rechtsstreit zugrundeliegende Thematik geschaffen werden soll. ${ }^{5}$

Ein zentraler Befund der Forschung zu Rechtsmobilisierung lautet: Gerichtsverfahren allein führen selten zur Veränderung politischer Entscheidungen und Inhalte, sondern sind meist eine Ergänzung anderer Strategien wie Lobbying oder öffentlichem Protest (Fuchs 2019, S. 245-246; McCann 2016, S. 4). Das Recht und die Ansprüche, die sich daraus ableiten lassen, als Vehikel für die Formulierung politischer Anliegen muss insofern als eine Ressource von unbestimmtem Wert angesehen werden. Diese Ressource kann jedoch zur politischen Mobilisierung eingesetzt werden (Scheingold 2004, S. 6-8). Mit politischer Mobilisierung lässt sich die Aktivierung von Individuen als Bürger und Inhaber von Rechten und die aktive Ermunterung zur Beteiligung (an kollektivem Handeln) verstehen (Bomberg 2012). Mobilisierung umfasst dabei auch den Aufbau von organisatorischen Strukturen, die kollektives Handeln auch über längere Dauer ermöglichen (Rucht 2008; Epp 2008b). ${ }^{6}$

Verfassungsbeschwerden, die der Mobilisierung für politische, gesamtgesellschaftliche Anliegen dienen, lassen sich als Phänomen kollektiven Handelns fassen und weisen insofern über den Charakter eines individuellen Rechtsbehelfs hinaus. In der gezielten Investition in Öffentlichkeitsarbeit und Organisation von Beteiligung liegt das entscheidende Alleinstellungsmerkmal sog. Massenverfassungsbeschwerden (Schreier 2011, S. 50-60). Diese zeichnen sich dadurch aus, dass sie

- von zivilgesellschaftlichen Organisationen und Gruppen angestoßen und mit einem öffentlichen Aufruf zur Unterstützung verknüpft werden,

- ein Anliegen verfolgen, das über die individuellen Interessen der Beschwerdeführer hinausweist und als gesamtgesellschaftlich relevant dargestellt wird,

- in ein breites Repertoire an Aktivitäten (z. B. Demonstrationen, Diskussionsveranstaltungen, Aufklärungskampagnen, Petitionen) eingebettet sind,

- über die Anrufung des Verfassungsgerichts darauf abzielen, für politische Anliegen breitere öffentliche Resonanz zu schaffen und politischen Gegendruck aufzubauen.

Massenverfassungsbeschwerden können mithin als öffentlichkeitswirksamer Versuch gewertet werden, Bürger für die Einforderung ihrer Rechte und für die Ausübung von Druck auf politische Entscheidungsträger zu mobilisieren. So gesehen erfüllen diese Verfassungsbeschwerden zwei Funktionen, die gemeinhin mit Outsidelobbying 7 in Verbindung gebracht werden (Kollman 1998, S. 25):

- die Bedeutung und Dringlichkeit eines Anliegens gegenüber politischen Entscheidungsträgern sollen signalisiert und betont werden;

\footnotetext{
5 Beispielhaft findet sich eine solche Sichtweise bei der Gesellschaft für Freiheitsrechte, die auf die Verteidigung und Durchsetzung von Menschen- und Grundrechten spezialisiert ist. https://freiheitsrechte.org/ strategische-klagen/ (letzter Zugriff am 26.10.2019).

6 Scheingold (2004, S. 131) sieht in Mobilisierung einen zweigleisigen Prozess aus der Aktivierung bislang apathischer Bürger und der Organisation von zu politischem Handeln fähiger Gruppen.

7 Kollman (1998, S. 3) definiert „outside lobbying“ als ,attempts by interest group leaders to mobilize citizens outside the policymaking community to contact or pressure public officials inside the policymaking community“.
} 
- der Konflikt soll ausgeweitet und in eine andere Arena verlagert werden, in der sich Berücksichtigungszwänge schaffen und Anliegen u. U. einfacher durchsetzen lassen.

$\mathrm{Zu}$ betonen ist, dass nicht das Policymotiv ausschlaggebend für die Differenzierung ist. Ein Beispiel ist die im Kern „klientelorientierte Verfassungsbeschwerde“ (Schreier 2011, S. 59) der privaten Krankenversicherer gegen das sog. GKV-Wettbewerbsstärkungsgesetz, das die Unternehmen wegen des Zwangs zur Einführung eines vom Krankheitsrisiko unabhängigen Basistarifs als Zerstörung ihres Geschäftsmodells anprangerten. Entscheidend ist vielmehr die Frage, ob die Beschwerdeführer die Unterstützung abgrenzbarer Gruppen oder prinzipiell der gesamten Bevölkerung suchen. Wo mit der Verfassungsbeschwerde der Erhalt des Status quo zur Absicherung bestehender, mit materiellen Interessen verknüpfter Rechtspositionen angestrebt wird, ist zu große Öffentlichkeit womöglich sogar kontraproduktiv. Demgegenüber ist Öffentlichkeit und eine breite Mobilisierung dort entscheidend, wo das Ziel eine Veränderung des Status quo, die Erschließung neuer Rechtspositionen und die Bekundung von Wertüberzeugungen ist.

Als Organ, das der politischen Einflussnahme bewusst entzogen ist, wird das Verfassungsgericht in der Regel alles tun, um den Eindruck zu vermeiden, es lasse sich von anderen als verfassungsrechtlichen Erwägungen leiten und etwa von einer massenhaften Zahl an Klägern beeindrucken (Aden 2014, S. 246). Zur kollektiven Rechtsmobilisierung eingesetzte Verfassungsbeschwerden können vielmehr das handfeste Risiko bergen, dass der Urteilsspruch die Einschätzung der politischen Entscheidungsträger in Regierung und Parlament verfassungsrechtlich absegnet und damit den Klägern auch für zukünftige Mobilisierungsversuche jeglichen Wind aus den Segeln nimmt. Auch lässt sich nicht jedes politische Anliegen als verfassungsrechtliches Problem umdeuten, das einer Klärung des Verfassungsgerichts bedarf. Überdies besteht für Interessengruppen, die über ein Verbandsklagerecht verfügen, ein geringerer Anreiz, den Umweg über Individualbeschwerden vor dem BVerfG zu nehmen, um ihre politischen Anliegen auf dem Rechtsweg zu erreichen (Aden 2014, S. 245). Schließlich können ideologische und kulturelle Prädispositionen bestimmte Gruppen davon abhalten, eine gerichtsorientierte Mobilisierungsstrategie zu verfolgen (Hilson 2002, S. 241; Pralle 2003, S. 238-243), etwa weil diese den sprichwörtlichen langen Atem braucht oder weil diese Gruppen eine kollektive Identität aus nichtkonventionellen, disruptiven Formen politischer Beteiligung beziehen (Rucht 2001, S. 130).

\section{Theoretischer Rahmen und Hypothesen}

\subsection{Organisationstypus und die Wahl von Taktiken und Strategien}

Die Interessengruppen- und Bewegungsforschung liefert wichtige Erklärungsansätze dafür, welche Formen organisierter Interessen am meisten durch die Externalisierung von Konflikten zu gewinnen haben (Beyers 2008; Dür und Mateo 2013; Kollman 
1998). Grundlegend lassen sich organisierte Interessen danach unterscheiden, ob es sich:

a) um Organisationen mit individueller Mitgliedschaft handelt, d.h. um Interessengruppen im engeren Sinne (Jordan et al. 2004); und

b) ob diese Organisation spezifische Interessen ihrer Mitglieder repräsentieren (,,sectional groups") oder diffuse Interessen eines breiteren Segments der Bevölkerung vertreten („,cause groups“) (Grant 2000, S. 18; Klüver 2013, S. 119).

In Anlehnung an Schattschneider (1975 [1960], S. 39-40) sind in erster Linie zivilgesellschaftliche Interessengruppen (,cause groups“) auf Konfliktexpansion angewiesen, um Einfluss auf politische Entscheidungsträger zu nehmen. Aus der Perspektive einer tauschbasierten Zugangstheorie (Bouwen 2002) können ,sectional groups"ein wichtiges Gut bereitstellen, das ihnen Zugang zu politischen Entscheidungsträgern verschafft: fachpolitische Expertise. Der direkte Zugang zu Entscheidungsträgern mindert den Anreiz zu öffentlichkeitswirksamen, konfrontativen Strategien der Interessenvermittlung. Ein zweites Argument bezieht sich auf die Integrationsfähigkeit und den Organisationserhalt von Gruppen - einem alten Thema kollektiver Handlungstheorien (Olson 1971). Die interne Integrationskraft von ,sectional groups"und ihr wesentliches Instrument der Mitgliedergewinnung beruht auf materiellen selektiven Anreizen (z. B. Serviceleistungen und Vergünstigungen). Diese bleiben Mitgliedern vorbehalten, die sich an der Erbringung eines Kollektivguts beteiligen. Demgegenüber binden zivilgesellschaftliche Interessengruppen Mitglieder hauptsächlich über die Gewährung immaterieller selektiver Anreize an sich. Dies sind zum einen expressive Leistungen, die durch die Äußerung und Bekundung von Interessen und Werten eines Individuums oder einer Gruppe entstehen. Zum anderen erbringen Interessengruppen solidarische Leistungen, die im Wesentlichen durch Akte der Versammlung entstehen und sich auf das Zusammengehörigkeitsgefühl, die Idee gemeinsam geteilter Überzeugungen oder der Abgrenzung zu anderen sozialen Gruppen gründen (Salisbury 1969, S. 15-22).

Zivilgesellschaftliche Interessengruppen und Gewerkschaften setzen in besonderem Maße auf Strategien des Outside obbying. Wenngleich auch Unternehmen oder Industrieverbände bis zu einem gewissen Grad öffentlichkeitsorientierte Strategien nutzen, sind die konfliktorientiertesten Handlungen wie die Organisation von Petitionen oder die Beteiligung an Demonstrationen Akteuren vorbehalten (Kollman 1998, S. 52-56; Tresch und Fischer 2015), die für die Durchsetzung ihrer Ziele in erster Linie auf die Mobilisierung von Mitgliedern angewiesen sind. Bereits gut organisierte Gruppen für den Einsatz für kollektive Ziele rekrutieren zu können ist ein Schlüssel für rasche Mobilisierung (Oberschall 1973, S. 125). Aus der Bedeutung einer mobilisierungsfähigen Mitgliederbasis lässt sich eine erste Hypothese ableiten:

H1 Wenn Mitgliederorganisationen als Beschwerdeführer auftreten, geht die Verfassungsbeschwerde mit größerer Mobilisierung einher. 


\subsection{Verfassungsbeschwerden und Ressourcenmobilisierung}

Der „Clou“, aber auch die Herausforderung von Verfassungsbeschwerden, die zur politischen Mobilisierung genutzt werden, besteht darin, Unmut und Protest zu bündeln und in ein gegenüber bloßem „Druck von der Straße“ hochgradig formalisiertes Verfahren zu kanalisieren. Sozialer Konfliktaustrag vor Gericht ist angewiesen auf Ressourcen, die von Mitgliedern der Gesellschaft bereitgestellt und von Organisationen aggregiert werden müssen. Epp (2008b, S. 3) argumentiert, dass erst die Generierung materieller Ressourcen und der Aufbau einer gesellschaftlichen „Stützstruktur" das strukturelle Ungleichgewicht zugunsten finanz- und ressourcenstarker Unternehmen und wohlhabender Individuen vor Gericht kompensieren konnte. Selbst wo eine solche Stützstruktur vorhanden ist, ist jedoch davon auszugehen, dass das Ungleichgewicht in der Ressourcenausstattung fortbesteht: Denn zivilgesellschaftliche Interessengruppen arbeiten anders als Unternehmen für gewöhnlich nicht gewinnorientiert. Erfolg oder Misserfolg hängen daher entscheidend davon ab, ob es gelingt, Sympathisanten zu Aktivisten zu machen, die ihr Geld, ihre Zeit und ihre Expertise zur Unterstützung eines gesellschaftlichen Anliegens bereitstellen (McCarthy und Zald 1977, S. 1222). Entsprechend lässt sich folgende Annahme formulieren:

H2 Wenn Beschwerdeführer über eine geringe Ressourcenausstattung verfügen, geht die Verfassungsbeschwerde mit größerer Mobilisierung einher.

\subsection{Verfassungsbeschwerden und öffentliche Meinung}

Die Umwandlung der Verfassungsbeschwerde von einem individuellen Rechtsbehelf in ein Instrument politischer Mobilisierung macht es erforderlich, ,das politische Allgemeinwohlanliegen in eine parallele individuelle Rechtsposition zu transformieren" (Aden 2014, S. 245) - und zwar nicht irgendeine, sondern eine verfassungsrechtlich relevante, auf Grundrechte gestützte Rechtsposition. Ein erster Prüfstein ist aus Sicht der Beschwerdeführer die Frage, inwieweit das Anliegen überhaupt von der Bevölkerung unterstützt wird. Eine Forderung, die von der Bevölkerung bereits ohne gezielte Mobilisierung mehrheitlich unterstützt wird, kann gegenüber dem BVerfG eher als dringlich und mehrheitsfähig dargestellt werden als eine, die nur bei einer Minderheit Zuspruch findet und insofern von politischen Kontrahenten leicht als partikulares Interesse diskreditiert werden kann. Über den relativen Erfolg erhöhter, medial vermittelter öffentlicher Aufmerksamkeit hinaus ist die Berücksichtigung des gesellschaftlichen Meinungsklimas aus Sicht der Kläger auch unmittelbar von Belang. So zeigen Sternberg et al. (2015) für Normenkontrollanträge der parlamentarischen Opposition, dass ein Urteilsspruch im Sinne der Opposition umso wahrscheinlicher ist, je größer die Unterstützung für die zugrundeliegenden Anliegen in der Bevölkerung ausfällt. Daraus ergibt sich eine dritte Annahme:

H3 Wenn eine Mehrheit in der Bevölkerung das Anliegen der Beschwerdeführer unterstützt, geht die Verfassungsbeschwerde mit größerer Mobilisierung einher. 


\subsection{Verfassungsbeschwerden als mediales Ereignis}

Verfassungsbeschwerden, in denen es den Klägern jenseits des objektiven Erfolgs um die gezielte Aktivierung der Öffentlichkeit und die Schaffung von Akzeptanz für die eigene Position geht, werden auch als Form der „Litigation-PR“ eingestuft (Köhler 2012, S. 209-212). Berücksichtigung und Erwähnung in den Medien gilt als ein wichtiges Instrument von Interessengruppen, um ihre Haltung zu spezifischen politischen Entscheidungen und Ergebnissen zu signalisieren, ihre Anhängerschaft zu mobilisieren und über eine erhöhte öffentliche Aufmerksamkeit für politische Entscheidungsprozesse Kontrolle über Entscheidungsträger auszuüben. Interessengruppen, deren Anliegen sich gegen den politischen Status quo richten bzw. die gegen spezifische Policyentscheidungen opponieren, gewinnen signifikant mehr mediale Aufmerksamkeit als solche, deren Position unklar bleibt bzw. die sich eher wohlwollend zu politischen Entscheidungen verhalten (Bruycker und Beyers 2015). Zugleich sind in erster Linie zivilgesellschaftliche Interessengruppen darauf angewiesen, dass die Medien ihre Anliegen auf die Agenda setzen und ins öffentliche Bewusstsein rücken, denn sie verfügen im Allgemeinen über zu geringe Ressourcen, als dass sie Outsidelobbying ganz ,auf eigene Rechnung“ betreiben könnten (McCarthy und Zald 1977, S. 1229).

H4 Wenn der zugrundeliegende Streitgegenstand der Beschwerde ein hohes Maß an medialer Berichterstattung generiert, geht die Verfassungsbeschwerde mit größerer Mobilisierung einher.

\subsection{Rechtssatz- vs. Urteilsverfassungsbeschwerden}

Verfassungsbeschwerden können sich gegen jegliche Akte der öffentlichen Gewalt richten. $\mathrm{Zu}$ differenzieren ist zwischen Urteilsverfassungsbeschwerden, bei denen Gerichtsurteile angefochten werden, und Rechtssatzverfassungsbeschwerden, bei denen eine Grundrechtsverletzung durch hoheitliche Rechtsakte wie Gesetze, Rechtsverordnungen oder die Zustimmung zu einem völkerrechtlichen Vertrag geltend gemacht wird. Da etwa gegen Parlamentsgesetze der Rechtsweg nicht offensteht, entfällt ausnahmsweise auch das Gebot der Rechtswegerschöpfung. Dies ist gerade für zivilgesellschaftliche Interessengruppen ein strategisch elementarer Faktor. Erstens erspart es der klagenden Organisation Anwalts- und Gerichtskosten, wenn sie nicht den gesamten Instanzenzug durchlaufen muss. Zweitens wird ein Verfahren so erheblich abgekürzt. ${ }^{8}$ Die Aufmerksamkeit für ein Anliegen und damit die Mobilisierung lässt sich über einen längeren Zeitraum kaum aufrechterhalten. Als letzte Annahme lässt sich daher formulieren:

H5 Wenn sich Verfassungsbeschwerden gegen Gesetze und andere hoheitliche Rechtsakte wenden, gehen sie mit größerer Mobilisierung einher.

\footnotetext{
8 In den hier untersuchten Fällen betrug die durchschnittliche Verfahrensdauer bei Urteilsverfassungsbeschwerden 1442 Tage gegenüber 1042 Tagen für Rechtssatzverfassungsbeschwerden.
} 


\section{Verfassungsbeschwerden von Interessengruppen: Daten und Methode}

\subsection{Fallauswahl}

Die nachfolgende Analyse basiert auf 35 Verfassungsbeschwerden zu 19 distinkten Verfahren, über die das BVerfG im Zeitraum 2000 bis 2017 in mündlicher Verhandlung entschieden hat. Ausdrücklich unberücksichtigt bleiben alle Entscheidungen, die lediglich als Beschluss und somit ohne mündliche Verhandlung ergingen. Die Eingrenzung der Fallauswahl auf Urteile ist einerseits theoretischen, andererseits forschungspragmatischen Erwägungen geschuldet. Theoretisch sind Urteile deshalb interessanter, weil sie anders als Beschlüsse aufgrund mündlicher Verhandlung ergehen (Pestalozza 1991, S. 60). Damit kann von vornherein ein höherer medialer Stellenwert unterstellt werden (Krehbiel 2016, S. 991). Überhaupt ist es nahezu unmöglich, ohne die mit einer mündlichen Verhandlung verbundene mediale Berichterstattung nähere Details über die Beschwerdeführer oder die Motivation einer Verfassungsbeschwerde zu erfahren. Das methodisch bevorzugte Verfahren, die Fallauswahl auf alle beim BVerfG eingehenden Rechtssachen zu gründen, bleibt aus datenschutzrechtlichen Gründen verwehrt. ${ }^{9}$ Die hier untersuchten Verfassungsbeschwerden sind mithin das Produkt eines mehrstufigen Selektionsprozesses:

1. Erstens werden nur Verfassungsbeschwerden untersucht, die überhaupt zur Entscheidung angenommen worden sind. Damit bleibt ein „Friedhof der Fälle“ (Fuchs 2019, S. 248) ausgeblendet. Im Untersuchungszeitraum lag die Erfolgsquote bei gerade einmal 2,0 Prozent.

2. Zweitens werden nur Verfassungsbeschwerden berücksichtigt, die als Urteil ergangen sind. Wie aus Tab. 1 hervorgeht, beträgt der Anteil von Urteilen an allen Entscheidungen im Untersuchungszeitraum pro Jahr nie mehr als 3,4 Prozent.

3. Drittens beschränkt sich die Analyse auf Beschwerden, in denen die Beschwerdeführer entweder organisierte Interessen (Verbände, Unternehmen, Rundfunkanstalten, Religionsgemeinschaften) sind oder natürliche Personen die Beschwerde nicht als Privatperson, sondern stellvertretend für eine Interessengruppe einlegten. Damit reduziert sich das Sample an Urteilen im Zeitraum 2000 bis 2017 von 145 auf 34 Verfassungsbeschwerden. Es ist ausdrücklich zu betonen, dass die Selektionsmechanismen die externe Validität der Ergebnisse erheblich einschränken. Die Reichweite der Aussage gilt allenfalls für Beschwerden, die zur mündlichen Verhandlung angenommen worden sind, und nicht für die Hauptlast der Verfahren vor dem BVerfG. Diesem Sachverhalt trägt auch die Methode der Datenauswertung Rechnung.

\footnotetext{
9 Eine entsprechende Anfrage an das BVerfG vom 16.10.2017 verweist sowohl auf die Abwägung zwischen den schutzwürdigen Interessen der Verfahrensbeteiligten an der Geheimhaltung von sachbezogenen Daten und dem berechtigten Interesse Dritter an der Einsichtnahme in die Vorgänge z. B. zu Forschungszwecken.
} 
Tab. 1 Verfassungsbeschwerden pro Jahr nach Form der Entscheidung. (Quelle: Bundesverfassungsgericht)

\begin{tabular}{lll}
\hline Jahr & Beschlüsse & Urteile (Anteil in Prozent) \\
\hline 2000 & 346 & $6(1,7)$ \\
2001 & 354 & $7(1,9)$ \\
2002 & 360 & $3(0,8)$ \\
2003 & 263 & $6(2,2)$ \\
2004 & 276 & $9(3,2)$ \\
2005 & 228 & $8(3,4)$ \\
2006 & 286 & $5(1,7)$ \\
2007 & 217 & $5(2,3)$ \\
2008 & 332 & $4(1,2)$ \\
2009 & 309 & $3(1,0)$ \\
2010 & 289 & $1(0,3)$ \\
2011 & 269 & $3(1,1)$ \\
2012 & 230 & $3(1,3)$ \\
2013 & 166 & $3(1,8)$ \\
2014 & 191 & $3(1,5)$ \\
2015 & 275 & $0(0,0)$ \\
2016 & 298 & $6(2,0)$ \\
2017 & 294 & $2(0,7)$ \\
\hline
\end{tabular}

\subsection{Fuzzy-set-QCA und konfigurationale Kausalität}

Qualitative Comparative Analysis (QCA) ist ein maßgeblich auf Mengentheorie und formaler Logik basierendes Verfahren. QCA versteht sich als Mittelweg zwischen variablen- und fallorientierten Forschungszugängen und eignet sich für mittlere Fallzahlen, bei denen eine auf statistischen Verfahren beruhende Analyse mit hohen Schätzfehlern zu kämpfen hätte. Abgesehen davon ist die wichtigste Rechtfertigung für die Wahl dieser Methode die Annahme konfigurationaler Kausalität (Schneider und Wagemann 2013, S. 12): Im Gegensatz zu inferenzstatistischen Verfahren beruht QCA auf der Erwartung, dass erst das Zusammenwirken von Bedingungen ein Outcome erklärt. Zudem liegt QCA nicht die Idee zugrunde, von einer Stichprobe auf die gesamte Population zu schließen (Schneider und Wagemann 2009, S. 403).

In QCA werden Fälle gemäß ihrer Mitgliedschaft in bestimmten Bedingungsmengen und in einer Outcomemenge charakterisiert. Fuzzy-set-QCA erlaubt im Gegensatz zu Crisp-set-QCA die Zuordnung von Mengenmitgliedschaftswerten in einem Intervall von 0 bis 1 und ermöglicht somit neben einer kategorialen Differenzierung eine graduelle Abstufung: Eine Bedingung kann mehr oder weniger vorhanden sein. Herzstück der QCA ist die Ermittlung von Mengenbeziehungen zwischen Outcome und (kausaler) Bedingung. Eine notwendige Bedingung liegt vor, wenn das Outcome nie unabhängig von der Bedingung auftritt. Von einer hinreichenden Bedingung spricht man, wenn das Vorliegen der Bedingung das Auftreten des Outcomes garantiert. Da es in der Praxis selten deterministische Zusammenhänge dieser Art gibt, hat QCA zwei spezifische „Schätzgütemaße“ eingeführt, die Abstufungen ermöglichen. Die Konsistenz ist ein Maß dafür, bis zu welchem Grad eine Untermengenbeziehung vorliegt. Die Abdeckung teilt uns die Größenverhältnisse der Outcome- und 
Bedingungsmenge mit. Sie ist somit ein Maß für die empirische Relevanz kausaler Bedingungen und Konfigurationen.

\subsection{Daten und Kalibrierung}

\subsubsection{Outcome}

Daten zu den Verfassungsbeschwerden und den Urteilen entstammen der Datenbank auf der Webseite des BVerfG. Die Beobachtungseinheit ist ein Antrag, wobei das BVerfG regelmäßig mehrere Anträge zu einer Entscheidung zusammenzieht (Sternberg et al. 2015, S. 579). Das zu erklärende Outcome ist das Ausmaß politischer Mobilisierung. Diese Einordnung erfordert substanzielles theoretisches Hintergrundwissen zum Hintergrund der jeweiligen Klagen sowie zur Identität der Beschwerdeführer (die aus den Klageschriften i.d.R. nicht hervorgeht). ${ }^{10}$ Ausgangspunkt der Kalibrierung ist die weiter oben umrissene Typologie von Schreier (2011). Entsprechend wurde überprüft, ob die Beschwerdeführer öffentlich zur Unterstützung der Klage aufriefen und inwieweit die Verfassungsbeschwerden durch Protestaktionen und Informationskampagnen begleitet wurden. Außerdem wurde analysiert, inwieweit das Anliegen als gemeinwohlorientiert bzw. von öffentlichem Interesse dargestellt wurde. Zu diesem Zweck wurde eine Medienanalyse in drei deutschen Qualitätstageszeitungen (Süddeutsche Zeitung, Frankfurter Allgemeine Zeitung, taz), dem Onlinenachrichtenmagazin SPIEGEL Online, auf der Datenbank Nexis sowie auf den Homepages der Beschwerdeführer durchgeführt. Zur Erfassung der Reichweite der Mobilisierung wurde außerdem auf Basis der Urteilsschriften ermittelt, wie viele Beschwerdeführer hinter der Verfassungsbeschwerde stehen und wie viele Verfassungsbeschwerden zu einer Entscheidung zusammengefasst wurden. Die Kalibrierung der Outcomemenge erfolgte qualitativ gemäß folgender Entscheidungsregeln: Für Beschwerden, die sämtliche Kriterien einer Massenverfassungsbeschwerde erfüllen, wurde der Fuzzy-Wert 1 (d.h. vollständige Mitgliedschaft) vergeben. Das trifft beispielsweise auf die von Mehr Demokratie initiierte Verfassungsbeschwerde gegen den Europäischen Stabilitätsmechanismus (ESM) und Fiskalpakt zu, welche als Verstoß gegen das Demokratieprinzip und eine Aushöhlung parlamentarischer Haushaltsrechte dargestellt wurde, die das Wahlrecht ins Leere laufen lasse. Eine Reihe von Verfassungsbeschwerden erfüllen manche, aber nicht alle Kriterien und stellen insoweit graduelle Abstufungen einer „Massenverfassungsbeschwerde“ dar. So gab es bei der Verfassungsbeschwerde des Landesverbands Nordrhein-Westfalen des Bundes für Umwelt und Naturschutz (BUND), der gegen die Zwangsenteignung einer Obstwiese auf dem Gebiet des Braunkohletagebaus Garzweiler II zunächst erfolglos bis vor das Bundesverwaltungsgericht gezogen war, zwar keinen Aufruf zur Unterstützung der Beschwerde selbst, wohl aber eine Informationskampagne, mit der die Verfassungsbeschwerde als Widerstand gegen den klimaschädlichen Braunkohletagebau und als Verteidigung des „Rechts auf Heimat“ gegenüber privatwirt-

10 Die Identität der Beschwerdeführer wurde mithilfe einer Medienanalyse zu den Verfassungsbeschwerden sowie - wo dies zu keinem Ergebnis führte - durch die Auswertung der Datenbank zu Drucksachen und Plenarprotokollen des Deutschen Bundestags - ermittelt (www.pdok.bundestag.de). 
schaftlichen Profitinteressen dargestellt wurde. Demgegenüber sind die Verfassungsbeschwerden, die Unternehmen oder kleinere Gruppen von Einzelpersonen gegen Gerichtsurteile einlegen, als Gegenpol von Verfassungsbeschwerden mit breiter Mobilisierungswirkung einzuordnen. ${ }^{11}$

\subsubsection{Bedingungen}

Als Bedingungen werden zwei Gruppen von Einflussgrößen herangezogen: Einerseits akteursbezogene Eigenschaften, andererseits kontextspezifische Charakteristika. $\mathrm{Zu}$ den akteursbezogenen Größen sind zu zählen:

- Organisation mit individueller Mitgliedschaft (INDMITGL): In Anlehnung an Berkhout et al. (2015) lässt sich unterscheiden zwischen Organisationen, in denen natürliche Personen Mitglieder sein können, und Organisationen, die keine Mitgliederorganisationen in diesem Sinne sind (z. B. Unternehmen oder Gebietskörperschaften). Innerhalb der ersten Gruppe ist ferner zu differenzieren zwischen solchen Organisationen, in denen ordentliche Mitglieder das Führungspersonal wählen und qua Satzung über Mitbestimmungsrechte verfügen, und jenen, in denen die Mitgliedschaft auf die Zahlung von Beiträgen oder Spenden begrenzt ist.

- Ressourcen (RESS): Für die Operationalisierung der Ressourcen wurde ein additiver Index gebildet. Als erster Indikator geht die Anzahl von hauptamtlichen Mitarbeitern ein, die mit Lobbying betraut sind. Dieses Maß ist am ehesten über unterschiedliche Organisationstypen hinweg vergleichbar (Kollman 1998, S. 53-54). Da Daten für die Bundesebene nicht oder nur rudimentär vorliegen, kommt als Proxy lediglich die Anzahl an Vollzeitäquivalenten, die für die jeweiligen Interessengruppen in Brüssel tätig sind, in Frage. Diese Angabe findet sich im EUTransparenzregister bzw. in den Daten des Portals Lobbyfacts. ${ }^{12}$ Als zweiter Indikator fließt das Budget ein, das den jeweiligen Interessengruppen für Lobbying zur Verfügung steht. Wenngleich diese Angaben auf freiwilligen Selbstauskünften bestehen und sich ebenfalls auf Lobbying in Brüssel beziehen, sind sie ein weithin verfügbares Maß für Ressourcenstärke, das über unterschiedliche Akteurstypen hinweg vergleichbar ist. Als dritter Indikator geht die Anzahl der Hausausweise für den Deutschen Bundestag ein. Diese Angaben entstammen zwei Datensätzen der Lobby-Watchdog-Organisation abgeordnetenwatch.de. ${ }^{13}$ Als vierter Indikator geht die Größe der Belegschaft in den Index ein. Wie Mahoney (2007, S. 41) anführt, ist der Personalbestand ein geeignetes Maß für die finanziellen Ressourcen, die notwendig sind, um überhaupt eine Hauptstadtrepräsentanz aufzubauen und zu unterhalten. Diese Angaben wurden den Homepages der Unternehmen und

11 Die Entscheidungsregeln hinter der Kalibrierung des Outcomes und der Bedingungen werden detailliert im Anhang in Tab. 5 und 6 beschrieben.

12 https://lobbyfacts.eu/ (zuletzt abgerufen am 26.10.2019). Wenig bekannte Akteure - zumeist kleine und mittelständische Unternehmen - haben erwartungsgemäß keine Einträge, und es ist plausibel, dass diese auch keine speziell mit Lobbying befassten Mitarbeiter beschäftigen.

13 Während für nichtverbandliche organisierte Interessen (Unternehmen, Anwaltskanzleien) Angaben aus dem Jahr 2015 verfügbar sind, stammen die Angaben zu Verbänden aus dem Jahr 2017. 
Verbände oder öffentlich zugänglichen Quellen wie Wikipedia entnommen. Die einzelnen Indikatoren wurden mittels einer Faktorenanalyse auf zugrundeliegende Dimensionen überprüft. Die Gewichtungsfaktoren der einzelnen Indikatoren entsprechend den Faktorladungen auf der ersten Dimension (Döring und Bortz 2016, S. 282). Der so gebildete Index wurde auf einen Wertebereich von 0 bis 1 standardisiert und mittels der indirekten Methode kalibriert (Schneider und Wagemann 2013, S. 35-36).

Als kontextspezifische Faktoren wurden folgende Bedingungen definiert:

- Der Umfang der Medienberichterstattung über den Streitgegenstand (MEDBER): Hierzu wurde auf Basis einer diktionärbasierten Suche die Treffer in der Datenbank Nexis ermittelt, gerechnet vom Datum der Einreichung der Beschwerde bis einen Tag vor der Urteilsverkündung. ${ }^{14}$ Da das Skalenniveau dieser Variablen intervallskaliert ist, erfolgte die Kalibrierung auf Basis der z-transformierten Werte mittels der direkten Methode.

- Die spezifische Unterstützung der Bevölkerung für die Position der Beschwerdeführer (UNTBEV): Diese wird auf Grundlage von Umfragedaten ermittelt. Das Vorgehen ist angelehnt an das von Sternberg et al. (2015). Zunächst wurden durch die Lektüre der Urteile und Pressemitteilungen des BVerfG sowie weiterer Presseberichte der zentrale Streit- und Entscheidungsgegenstand ermittelt. In einem zweiten Schritt wurde versucht, Umfragen zu ermitteln, welche die Meinung der Bevölkerung zu diesen Streitthemen abfragen und maximal 18 Monate vor dem Urteilsspruch durchgeführt wurden. Nur in einem Teil aller Fälle ist jedoch das Entscheidungsthema wie etwa bei der Frage der Verfassungsmäßigkeit des ESM und Fiskalpakts so eindeutig, dass hierzu eigens eine Umfrage durchgeführt wurde. Daher wurde in einem dritten Schritt versucht, das Entscheidungsthema und eine entsprechende Umfrage über eine Proxy-Frage zu verknüpfen. ${ }^{15}$ Anders als die bisherigen Bedingungen erfolgte die Kalibrierung der Fuzzy-Menge nicht computergestützt, sondern qualitativ. Die Fuzzy-Werte sind daher nicht stetig, sondern kategorial ausgeprägt.

- Die Art der Verfassungsbeschwerde (RSVB): Diese dichotom kalibrierte Bedingungsmenge nimmt den Wert 1 für Rechtssatzbeschwerden und den Wert 0 für Urteilsverfassungsbeschwerden an.

- Der Grad, zu dem Parteien in die Mobilisierung des Rechts eingebunden sind (PARTEI): Auch wenn diese Größe nicht expliziter Bestandteil der Hypothesen ist, wird sie hier berücksichtigt, um der Parallelität von durch Parteien angestrengte Klagen Rechnung zu tragen. Diese Menge wurde qualitativ kalibriert und ist dichotom. Ein Fuzzy-Wert von 1 wurde für Verfassungsbeschwerden vergeben, in denen entweder von Bundestagsfraktionen Organklagen angestrengt wurden und/ oder die Mitglieder der Bundestagsfraktionen geschlossen als Privatpersonen Verfassungsbeschwerde erhoben.

14 Siehe hierzu Tab. 7 im Anhang.

15 Die zugrundeliegenden Umfragen und Items sind im Anhang in Tab. 8 aufgelistet. 


\section{Analyse und Diskussion}

\subsection{Analyse des Outcomes}

Die Analyse beginnt mit der Überprüfung notwendiger Bedingungen. Da die Wirkung der einzelnen Bedingungen zunächst unbestimmt ist, werden stets die Bedingungen und ihre Negation untersucht. Eine Bedingung übertrifft den konventionellen Konsistenzmaßschwellenwert von 0,9: Der Status als Rechtssatzverfassungsbeschwerde $(R S V B)$ mit einem Wert von 0,95. Nahe an dem Schwellenwert liegt zudem die Abwesenheit einer hohen Ressourcenausstattung (ress) mit einem Konsistenzwert von $0,87 .{ }^{16}$ Die Abdeckung als notwendige Bedingung liegt in beiden Fällen oberhalb von 0,5 und fällt damit relativ hoch aus. Für die weitere Analyse ergeben sich hieraus wichtige Hinweise auf die Wirkungsrichtung der beiden Bedingungen für das Outcome.

Da sechs Bedingungen in die Analyse eingehen, ergeben sich $2^{6}=64$ Konfigurationen. Tatsächlich kommen aber nur 13 und damit nur rund ein Fünftel aller logisch möglichen Konfigurationen empirisch vor - eine für QCA eher typische Situation (Schneider und Wagemann 2013, S. 169). Die Wahrheitstafel in Tab. 2 gibt für jede Konfiguration (Zeile) die Konsistenz als hinreichende Bedingung für das Outcome $(M O B I L)$ an. Das Outcome tritt stets in Verbindung mit den Bedingungen INDMITGL und $R S V B$ auf: INDMITGL ${ }^{*} R S V B$ ist eine INUS-Bedingung. Mittels QCA lassen sich drei Lösungsgleichungen ermitteln, die unterschiedliche Annahmen über logische Rudimente basieren. Während die konservative Lösung keinerlei Annahmen über die direktionale Wirkung empirisch nicht beobachteter Konfigurationen macht, bezieht die sparsamste Lösung alle logischen Rudimente ein, die zu einer Reduktion der Komplexität der Lösungsterme beitragen. Die mittlere Lösung enthält nur vereinfachende Annahmen, die mit empirischem und theoretischem Wissen konform sind.

Für die Ermittlung der Lösungsgleichung wurden alle Konfigurationen einbezogen, die oberhalb eines in der Literatur gebräuchlichen Schwellenwertes von 0,8 liegen (Schneider und Wagemann 2013, S. 127). Die Interpretation der Lösungsgleichung beschränkt sich aus Platzgründen auf die mittlere Lösung. ${ }^{17}$ Aus der Analyse der notwendigen Bedingungen ress und RSVB und in Kenntnis der INUS-Bedingung $I N D M I T G L^{*} R S V B$ lassen sich begründbare Annahmen über die Wirkung der einzelnen Bedingungen formulieren. Hinreichend für das Outcome sollte demnach das Vorliegen eines Organisationstypus mit individueller Mitgliedschaft, die Abwesenheit hoher Ressourcenausstattung sowie der Status der Beschwerde als Rechtssatz sein. Gemäß den Hypothesen wird im Folgenden unterstellt, dass die übrigen drei

\footnotetext{
16 Die Tabelle mit den Konsistenzwerten für notwendige Bedingungen findet sich aus Platzgründen im Anhang (Tab. 9). In der nachstehenden Analyse wird eine Groß-/Kleinschreibweise verwendet: Großbuchstaben zeigen das Vorliegen einer Bedingung an, Kleinbuchstaben deren Abwesenheit.

17 Die sparsamste und die komplexe Lösung sind im Anhang in Tab. 10 und 11 aufgeführt.
} 
Tab. 2 Wahrheitstafel für das Outcome MOBIL

\begin{tabular}{|c|c|c|c|c|c|c|c|c|}
\hline \multicolumn{6}{|c|}{ Bedingungen } & \multicolumn{3}{|l|}{ Outcome } \\
\hline INDMITGL & RESS & MEDBER & UNTBEV & PARTEI & RSVB & MOBIL & Fallzahl & Konsistenz \\
\hline 1 & 0 & 0 & 1 & 0 & 1 & 1 & 6 & $0,975^{\mathrm{a}}$ \\
\hline 1 & 0 & 0 & 0 & 1 & 1 & 1 & 2 & $1,000^{\mathrm{a}}$ \\
\hline 1 & 0 & 1 & 1 & 1 & 1 & 1 & 2 & $1,000^{\mathrm{a}}$ \\
\hline 1 & 1 & 0 & 1 & 0 & 1 & 1 & 1 & $1,000^{\mathrm{a}}$ \\
\hline 1 & 0 & 0 & 1 & 1 & 1 & 1 & 1 & $1,000^{\mathrm{a}}$ \\
\hline 0 & 0 & 0 & 1 & 0 & 1 & 0 & 7 & 0,359 \\
\hline 0 & 0 & 0 & 0 & 0 & 0 & 0 & 4 & 0,000 \\
\hline 0 & 0 & 0 & 0 & 0 & 1 & 0 & 4 & 0,535 \\
\hline 0 & 1 & 1 & 0 & 0 & 1 & 0 & 3 & 0,406 \\
\hline 1 & 0 & 0 & 0 & 0 & 0 & 0 & 2 & 0,410 \\
\hline 0 & 0 & 0 & 1 & 0 & 0 & 0 & 1 & 0,000 \\
\hline 0 & 1 & 0 & 0 & 0 & 1 & 0 & 1 & 0,643 \\
\hline 0 & 1 & 0 & 1 & 0 & 1 & 0 & 1 & 0,549 \\
\hline
\end{tabular}

${ }^{a}$ Konfigurationen liegen oberhalb des Schwellenwertes von 0,80 als hinreichende Bedingung für das Outcome

Aus Platzgründen werden die logischen Rudimente nicht angeben

Bedingungen in ihrer Anwesenheit hinreichend für das Outcome sind. Dann reduziert sich die Lösungsgleichung auf:

\section{INDMITGL*UNTBEV*RSVB + INDMITGL*ress*PARTEI*RSVB $\rightarrow$ MOBIL}

In Worten ausgedrückt: hinreichend für eine breite Mobilisierung mittels einer Verfassungsbeschwerde ist

- sowohl die Kombination aus einer Mitgliederorganisation, deren Klage Unterstützung in der Bevölkerung findet und die sich gegen einen formalen Rechtsakt richtet,

- als auch die Kombination aus Mitgliederorganisation, die ressourcenschwach ist und die eine Verfassungsbeschwerde gegen ein formales Gesetz einlegt, gegen das parallel Parteien vor dem BVerfG klagen.

Die Lösungsgleichung erreicht mit 0,988 einen sehr hohen Konsistenzwert (s. Tab. 3). Dies veranschaulichen auch die XY-Plots in Abb. 1. Es gibt nur einen Fall, der unterhalb der Diagonalen liegt und damit die Annahme perfekter Konsistenz verletzt. Hierbei handelt es sich um die Verfassungsbeschwerde der evangelischen Kirche Berlin-Brandenburg gegen die Ladenöffnung an allen vier Adventssonntagen. Diese Konfiguration weist einen Mitgliedschaftswert von 0,8 in der Bedingungsmenge INDMITGL*UNTBEV*RSVB auf, während der Mitgliedschaftswert in der Outcomemenge mit 0,7 geringer ausfällt.

Zusammen umfassen die zwei Konfigurationen mehr als die Hälfte aller Fälle, in denen das Outcome auftritt. Hierbei ist jedoch die erste, weniger komplexe Konfiguration INDMITGL*UNTBEV*RSVB der wichtigere Pfad: Die alleinige Abdeckung ist mit 0,309 deutlich höher als jene für die Konfiguration IND- 
Tab. 3 Lösungsgleichung für das Outcome MOBILI Mittlere Lösung

\begin{tabular}{lllll}
\hline Lösungspfad & $\begin{array}{l}\text { Rohab- } \\
\text { deckung }\end{array}$ & $\begin{array}{l}\text { Alleinige } \\
\text { Abdeckung }\end{array}$ & $\begin{array}{l}\text { Konsis- } \\
\text { tenz }\end{array}$ & Exemplarische empirische Fälle \\
\hline $\begin{array}{l}\text { INDMITGL* } \\
\text { UNTBEV* }\end{array}$ & 0,487 & 0,309 & 0,987 & $\begin{array}{l}\text { Mehr Demokratie gegen ESM und Fiskal- } \\
\text { pakt; Gewerkschaften gegen Tarifeinheits- } \\
\text { gesetz }\end{array}$ \\
$\begin{array}{l}\text { RSVB } \\
\begin{array}{l}\text { INDMITGL* } \\
\text { ress*PARTEI* }\end{array}\end{array}$ & 0,237 & 0,059 & 1,000 & $\begin{array}{l}\text { Grimmenstein via Change,org und Cam- } \\
\text { pact/foodwatch/Mehr Demokratie gegen } \\
\text { RSVB }\end{array}$ \\
& & & $\begin{array}{l}\text { CETA; AK Vorrat gegen Vorratsdatenspei- } \\
\text { cherung; Mehr Demokratie gegen OMT- } \\
\text { Programm }\end{array}$ \\
\hline
\end{tabular}

Konsistenz der Lösungsgleichung: 0,988

Abdeckung der Lösungsgleichung: 0,546

MITGL*ress ${ }^{*} P A R T E I * R S V B(0,059)$. Dies ist auch daran zu erkennen, dass im linken XY-Plot mehr Fälle weiter oben rechts liegen, d.h. hohe Werte für die Mitgliedschaft in der Bedingungsmenge mit hohen Werten der Outcomemenge einhergehen. Die Lösungsgleichung offenbart Äquifinalität: Das Outcome tritt in Verbindung mit substanziell unterschiedlichen Konfigurationen auf, die sich allerdings in einem Punkt gleichen: Beide Lösungspfade enthalten die Bedingung INDMITGL*RSVB, die zugleich die sparsamste Lösung darstellt.

Betrachtet man die Fälle, die durch die beiden Lösungspfade beschrieben werden, so wird der Unterschied zwischen den Klägern und dem Kontext der Verfassungsbeschwerden deutlich. Der erste Lösungspfad umfasst für sich genommen eine heterogene Menge an Beschwerden: Zum einen die Beschwerden der Dienstleistungsgewerkschaft ver.di und kleinerer Spartengewerkschaften gegen das Tarifeinheitsgesetz sowie die von Mehr Demokratie angeführten Massenverfassungsbeschwerden gegen ESM und Fiskalpakt und gegen das OMT-Anleihekaufprogramm der Europäischen Zentralbank (EZB). Diese zeichnen sich jedoch bei allen Unterschieden dadurch aus, dass die Klagen mit einer hohen bis sehr hohen Unterstützung (mehr als 40 Prozent Zustimmung in der Bevölkerung für die Position der Beschwerdeführer) einhergehen. Der zweite Lösungspfad beschreibt die beiden Verfassungsbeschwerden gegen die Ratifizierung und vorläufige Anwendung des CETA sowie gegen die Vorratsdatenspeicherung. Diese Gruppe von Beschwerden repräsentierten die eingangs beschriebenen Massenverfassungsbeschwerden. Die Klagen der zweiten Gruppe heben sich nicht zuletzt dadurch von den anderen Klagen ab, dass Sie parallel durch teils mehrere Verfassungsbeschwerden ehemaliger und aktiver Abgeordneter sowie durch Verfassungsklagen von Parteien begleitet wurden..$^{18}$ Alle durch die Lösungsgleichung beschriebenen Fälle eint der Umstand, dass die Verfassungsbeschwerden mehrere tausend Beschwerdeführer mobilisierten und mit Protestaktionen und/ oder Petitionen einhergingen. Auffällig ist zudem, dass Mehr Demokratie an drei

\footnotetext{
18 Gegen die Vorratsdatenspeicherung klagten vor dem BVerfG eine Gruppe von FDP-Abgeordneten um die spätere Justizministerin Sabine Leutheusser-Schnarrenberger sowie die Abgeordneten der Bundestagsfraktion der Grünen, die darüber hinaus eine Organklage einreichte. Die Klage gegen CETA wurde durch eine Verfassungsbeschwerde der 63 Abgeordneten der LINKEN-Fraktion unterstützt.
} 
Abb. 1 XY-Plots der als hinreichend identifizierten Konfigurationen

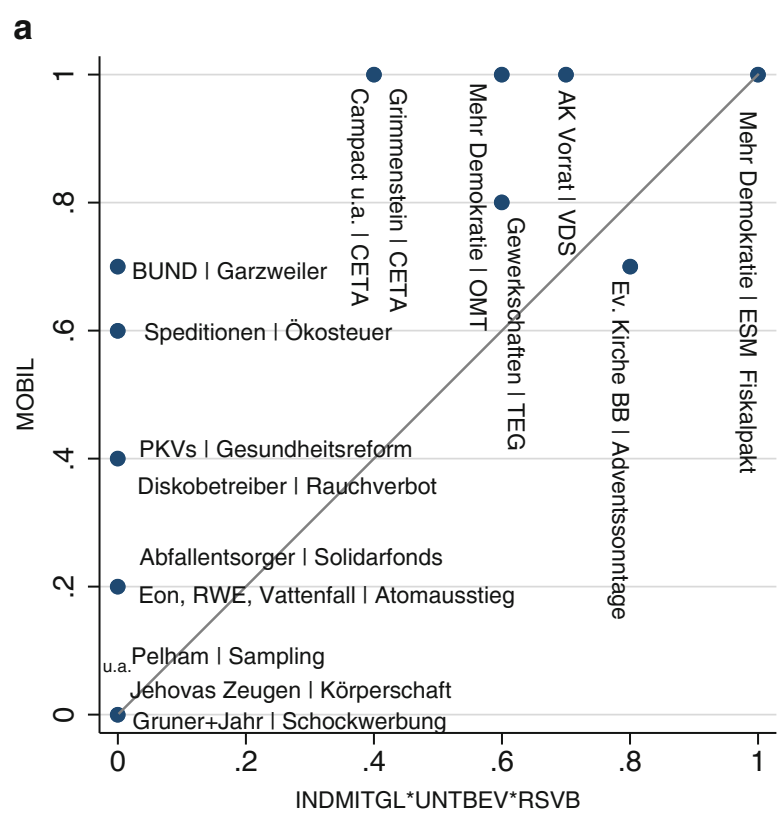

b

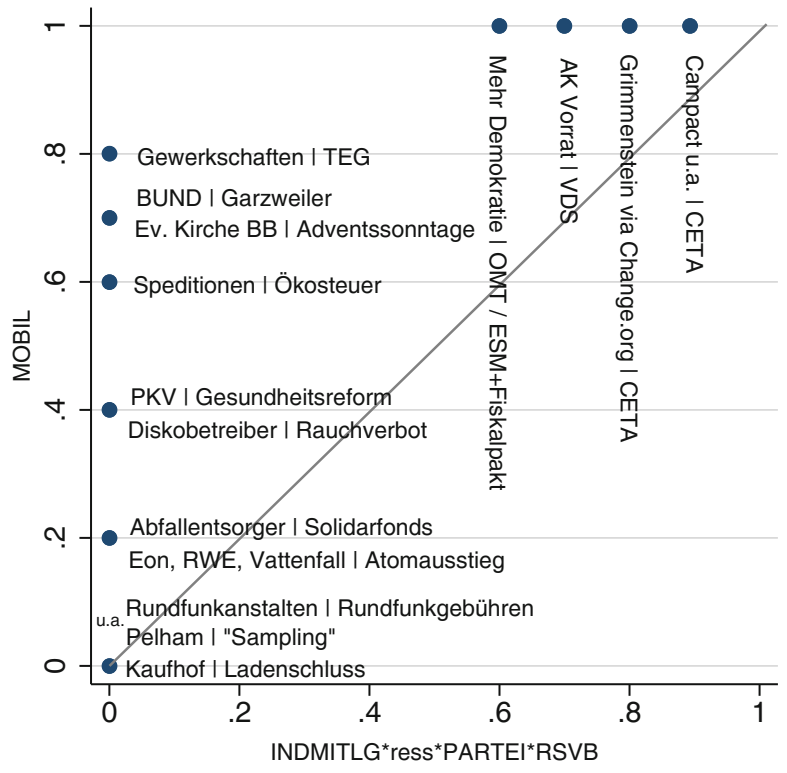

Massenverfassungsbeschwerden direkt beteiligt war. Die Organisation kann im hier untersuchten Sample als ,repeat player“ (Galanter 1974) gelten.

Insgesamt liefern die Ergebnisse starke Belege für H1 und H5: Der Charakter als individuelle Mitgliedschaftsorganisation und der Status als Rechtssatzverfassungsbeschwerde sind notwendige, aber nicht hinreichende Bestandteile einer hinreichenden, aber nicht notwendigen Bedingung für eine breite Mobilisierung. Mit Einschränkun- 
Tab. 4 Lösungsgleichungen für das Non-Outcome (mittlere Lösung)

\begin{tabular}{lllll}
\hline Lösungspfad & $\begin{array}{l}\text { Rohab- } \\
\text { deckung }\end{array}$ & $\begin{array}{l}\text { Alleinige } \\
\text { Abdeckung }\end{array}$ & $\begin{array}{l}\text { Konsis- } \\
\text { tenz }\end{array}$ & Exemplarische empirische Fälle \\
\hline $\begin{array}{l}\text { indmitg1* } \\
\text { ress*medber* } \\
\text { partei }\end{array}$ & 0,608 & 0,201 & 0,912 & $\begin{array}{l}\text { UCI gegen Filmabgabeförderung; Pel- } \\
\text { ham (,Sampling“); Gruner+Jahr gegen } \\
\text { Verbot von ,Schockwerbung“; Logistik- } \\
\text { unternehmen gegen Ökosteuer }\end{array}$ \\
$\begin{array}{l}\text { indmitgl*medber* } \\
\text { partei*RSVB }\end{array}$ & 0,454 & 0,027 & 0,853 & $\begin{array}{l}\text { Rundfunkanstalten gegen Berechnung } \\
\text { der Rundfunkgebühren }\end{array}$ \\
$\begin{array}{l}\text { indmitgl*RESS* } \\
\text { untbev*partei* }\end{array}$ & 0,183 & 0,111 & 0,948 & $\begin{array}{l}\text { Energiekonzerne gegen Atomausstieg } \\
\text { RSVB }\end{array}$ \\
\hline
\end{tabular}

Konsistenz der Lösungsgleichung: 0,906

Abdeckung der Lösungsgleichung: 0,766

gen kann auch $\mathrm{H} 2$ akzeptiert werden. Eine schwache Ressourcenausstattung (ress) ist für sich genommen zwar keine hinreichende, jedoch eine annähernd notwendige Bedingung für das Outcome. Drei der vier empirisch beobachteten Konfigurationen, die mit dem Outcome verknüpft sind, beinhalten die Bedingung, und in einem Pfad der Lösungsgleichung ist die Bedingung ebenfalls enthalten. Für H3, H4 und H5 fallen die Befunde hingegen ambivalenter aus. Die Unterstützung in der Bevölkerung erweist sich als Bestandteil der ersten Konfiguration, nicht aber der zweiten. Umgekehrt sind hohes mediales Interesse und parallel über Parteien organisierte Verfassungsklagen Bestandteile des zweiten Pfades, nicht aber des ersten. Die empirischen Ergebnisse sind robust gegenüber alternativen Operationalisierungen einzelner Bedingungen sowie gegenüber höherer Frequenzschwellenwerte für einzelne Konfigurationen. Multiple Regressionsanalysen liefern starke empirische Evidenz insbesondere für die Bedeutung von Rechtssatzverfassungsbeschwerden für das Ausmaß politischer Mobilisierung. ${ }^{19}$

\subsection{Analyse des Non-Outcomes}

Wie aus Tab. 8 im Anhang ersichtlich wird, ist eine einzige Bedingung konsistent notwendig für das Non-Outcome: Die Abwesenheit parallel von Parteien organisierter Verfassungsklagen (partei). Mit Blick auf die hinreichenden Konfigurationen lassen sich auf Basis der mittleren Lösung drei Pfade identifizieren, die hinreichend für einen geringen Mobilisierungsgrad sind (s. Tab. 4). ${ }^{20}$ In allen ist die als notwendig identifizierte Bedingung partei enthalten. Diese Konfigurationen repräsentieren sowohl sehr unterschiedliche Beschwerdeführer als auch sehr unterschiedliche Klagen: Während beispielsweise der Pfad mit der höchsten Einzelabdeckung indmit*ress*medber*partei die Klagen der Rundfunkanstalten gegen die Festsetzung

\footnotetext{
19 Die Ergebnisse der Robustheitstests finden sich im Anhang.

20 Die mittlere Lösung entspricht der komplexen Lösung unter der Annahme, dass die direktionale Wirkung der Bedingungen für das Non-Outcome unbestimmt ist. Dies ist in unserem Fall plausibel, da man nicht davon ausgehen kann, dass für das Non-Outcome gleichsam spiegelbildlich die komplementären Bedingungen hinreichend sind wie für das Outcome.
} 
der Rundfunkgebühren durch die Länder oder des ZDF und dreier Journalisten gegen die Ausspähung von Mobilfunkdaten zur Strafverfolgung repräsentieren, bildet der Pfad mit der höchsten Konsistenz indmitgl*RESS*untbev*partei*RSVB die Rechtssatzverfassungsbeschwerden ressourcenstarker Unternehmen wie privater Krankenversicherungen gegen die Gesundheitsreform oder der Energieversorger gegen den beschleunigten Atomausstieg infolge des Reaktorunglücks in Fukushima ab.

\section{Schlussbetrachtung und Ausblick}

Verfassungsbeschwerden sind bislang kaum theoretisch als Mobilisierungsinstrument gewürdigt worden. Dies ist erstaunlich, da selbst die Rechtswissenschaft der Verfassungsbeschwerde eine Mobilisierungs- und Partizipationsfunktion zubilligt (Gusy 2015; Zuck 2013). In Anknüpfung an die Literatur zur Rechtsmobilisierung und unter Rückgriff auf Theorien der Interessengruppen- und Bewegungsforschung ist dieser Beitrag der Frage nachgegangen, unter welchen Bedingungen Verfassungsbeschwerden breite Mobilisierung generieren.

Die Ergebnisse einer Fuzzy-set-Analyse von insgesamt 35 Verfassungsbeschwerden, die von organisierten Interessen eingebracht worden sind und über die nach mündlicher Verhandlung entschieden wurde, liefert eine Reihe erhellender Einsichten, die in vielen Belangen anschlussfähig an die bisherige Forschung sind. Als hinreichende Bedingung für das Outcome wurden zwei kausale Pfade identifiziert, die bei allen Unterschieden den Umstand teilen, dass sie von Organisationen mit individueller Mitgliedschaft eingebracht worden sind und sich gegen formale Rechtsakte wenden. Gerade im Vergleich zur Analyse des Non-Outcomes zeigt sich der Stellenwert individueller Mitgliedschaft: Unternehmen nutzen Verfassungsbeschwerden nicht als Mobilisierungsinstrument, sondern im Sinne eines konventionellen Grundrechtsbehelfs. Die Nutzung von Verfassungsbeschwerden als Taktik des Outsidelobbyings ist primär eine Domäne zivilgesellschaftlicher Interessengruppen. Dieser Befund ist kompatibel mit Ergebnissen aus der Interessengruppenforschung (Dür und Mateo 2013; Kollman 1998). Dass der Charakter als Rechtssatzverfassungsbeschwerde für sich genommen schon nahezu konsistent notwendig für den Status als Mobilisierungsinstrument ist, ist ebenfalls plausibel: Da gegen Parlamentsgesetze der Rechtsweg nicht offensteht, entfällt auch das Subsidiaritätsprinzip. Dies erst schafft die Voraussetzung für die Erhebung einer Verfassungsbeschwerde durch eine Vielzahl von Beschwerdeführern (Schreier 2011, S. 42-43). Demgegenüber ist einleuchtend, dass ressourcenstarke Unternehmen es sich viel eher leisten können, sich durch sämtliche Instanzen zu klagen, um zu ihrem Recht zu kommen.

Die Fuzzy-set-Analyse deckt zudem zwei unterschiedliche Pfade zum interessierenden Phänomen auf: Der erste Pfad, der durch die Konfiguration IND$M I T G L^{*} U N T B E V^{*} R S V B$ beschrieben wird, deutet auf die Relevanz öffentlicher Unterstützung im Vorfeld der Klage hin und ist kompatibel mit einer Sichtweise auf Verfassungsbeschwerden als Kulminationspunkt der Mobilisierung und des Konfliktaustrags. Demgegenüber beschreibt die Konfiguration IND$M I T G L *$ ress*MEDBER*PARTEI*RSVB einen Pfad, der eher mit der Sichtweise von Verfassungsbeschwerden als Mittel einer entschlossenen, aber strukturell unter- 
legenen Minderheit korrespondiert, die durch die Anrufung des BVerfG Unterstützung und Aufmerksamkeit für ihr Anliegen überhaupt erst generieren muss. Diese Perspektive passt eher ins Bild der Massenverfassungsbeschwerde, wie Schreier (2011) sie konzeptualisiert: Als Instrument der Mobilisierung von Unterstützung und Ressourcen, die auch für weitere Kampagnen und in anderen Arenen genutzt werden können. Sowohl die Ergebnisse der empirischen Analyse als auch die zeitliche Abfolge von Einreichung der Beschwerde und begleitenden Protestaktionen legen die Schlussfolgerung nahe, dass Verfassungsbeschwerden sich nur selten als Baustein anhaltender Mobilisierung und als Kristallisationspunkt für soziale Bewegungen nutzen lassen. Eine beispielhafte Ausnahme liefert der Fall der Vorratsdatenspeicherung, die nicht nur in Deutschland zivilgesellschaftlichen Protest zur Folge hatte (Thierse und Badanjak 2020). Nicht nur gewannen die Freiheitstatt-Angst-Demonstrationen erst nach der Einreichung der Beschwerde weiter an Zulauf; die Beschwerde war auch eingebettet in eine breitere Mobilisierung gegen staatliche Überwachungsmaßnahmen, die auch in weiteren und späteren Fällen verfassungsrechtlich angegriffen worden sind (Nocun und Breyer 2014).

Für die weitere Forschung ergeben sich eine Reihe von Desiderata und Anknüpfungspunkten. Erstens beruht die empirische Analyse dieses Beitrags ausschließlich auf Urteilen, die nur einen Bruchteil aller zur Entscheidung angenommenen Verfassungsbeschwerden ausmachen. Künftige Untersuchungen, die auch Beschlüsse systematisch berücksichtigen, können auf eine erheblich größere Fallzahl aufbauen und sind damit eher inferenzstatistischen Verfahren zugänglich. Zudem böte die Einbeziehung von Beschlüssen den Vorteil einer klareren Abgrenzung gewöhnlicher, von Individuen angestrengter Verfassungsbeschwerden von jenen, die hier als Phänomen kollektiver (Rechts-)Mobilisierung betrachtet wurden. Zweitens würde der kumulative Aufbau von Datenbeständen einen Beitrag dazu leisten, Größen wie die Ressourcenausstattung klagender Interessengruppen präziser und im Längsschnitt zu erfassen. Die vorliegende Arbeit musste mangels über Akteurstypen vergleichbarer und im Längsschnitt verfügbarer Daten für die nationale Ebene auf Proxy-Variablen zurückgreifen. Insbesondere für Organisationen, die nicht auf supranationaler Ebene aktiv sind, birgt dies die Gefahr von Messfehlern. Drittens sind perspektivisch ländervergleichende Analysen von Strategien der Rechtsmobilisierung wünschenswert. Damit würde nicht nur Variation in Bezug auf den individuellen Zugang zur Verfassungsgerichtsbarkeit erzeugt, sondern auch der Blick auf funktionale Äquivalente zu Verfassungsbeschwerden gerichtet. Viertens scheint ein relationaler, netzwerkanalytischer Zugang vielversprechend und geboten, um die Mobilisierung des Rechts durch Klagekollektive in zeitlicher und räumlicher Hinsicht nachzuzeichnen (Diani 2013). Nur so lässt sich schließlich klären, warum manche Interessengruppen gerichtsorientierte Strategien verfolgen, andere hingegen bewusst davon Abstand nehmen.

Danksagung Für zahlreiche konstruktive und hilfreiche Kommentare geht mein Dank an Katharina van Elten, Britta Rehder, Beate Kohler-Koch, Jan Beyers, Detlef Sack, Florian Spohr und Arndt Wonka sowie den zwei anonymen GutachterInnen. Für umfangreiche Zuarbeit bei der Datenerhebung danke ich Tim Vogt und Boris Bogdahn.

Funding Open Access funding provided by Projekt DEAL. 
Open Access Dieser Artikel wird unter der Creative Commons Namensnennung 4.0 International Lizenz veröffentlicht, welche die Nutzung, Vervielfältigung, Bearbeitung, Verbreitung und Wiedergabe in jeglichem Medium und Format erlaubt, sofern Sie den/die ursprünglichen Autor(en) und die Quelle ordnungsgemäß nennen, einen Link zur Creative Commons Lizenz beifügen und angeben, ob Änderungen vorgenommen wurden.

Die in diesem Artikel enthaltenen Bilder und sonstiges Drittmaterial unterliegen ebenfalls der genannten Creative Commons Lizenz, sofern sich aus der Abbildungslegende nichts anderes ergibt. Sofern das betreffende Material nicht unter der genannten Creative Commons Lizenz steht und die betreffende Handlung nicht nach gesetzlichen Vorschriften erlaubt ist, ist für die oben aufgeführten Weiterverwendungen des Materials die Einwilligung des jeweiligen Rechteinhabers einzuholen.

Weitere Details zur Lizenz entnehmen Sie bitte der Lizenzinformation auf http://creativecommons.org/ licenses/by/4.0/deed.de. 
Anhang

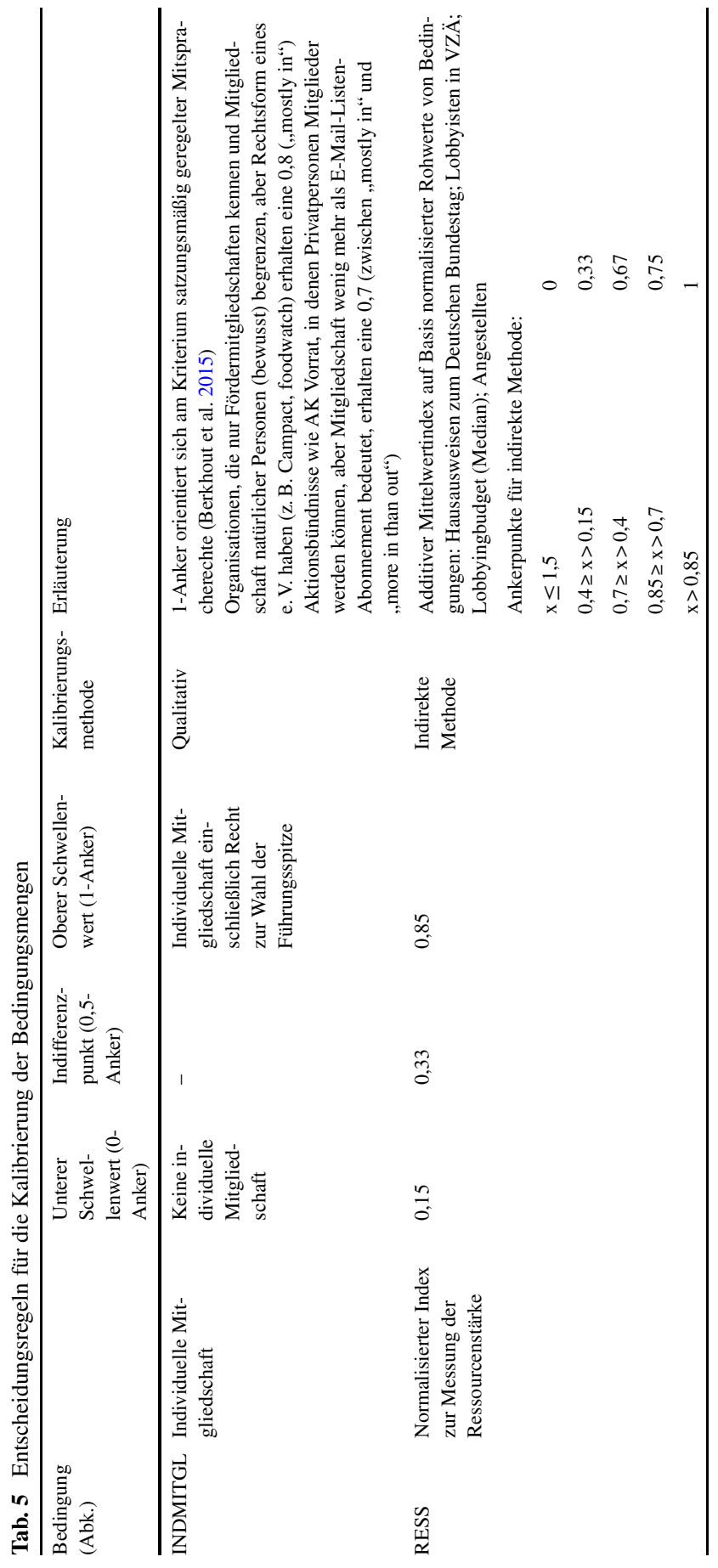




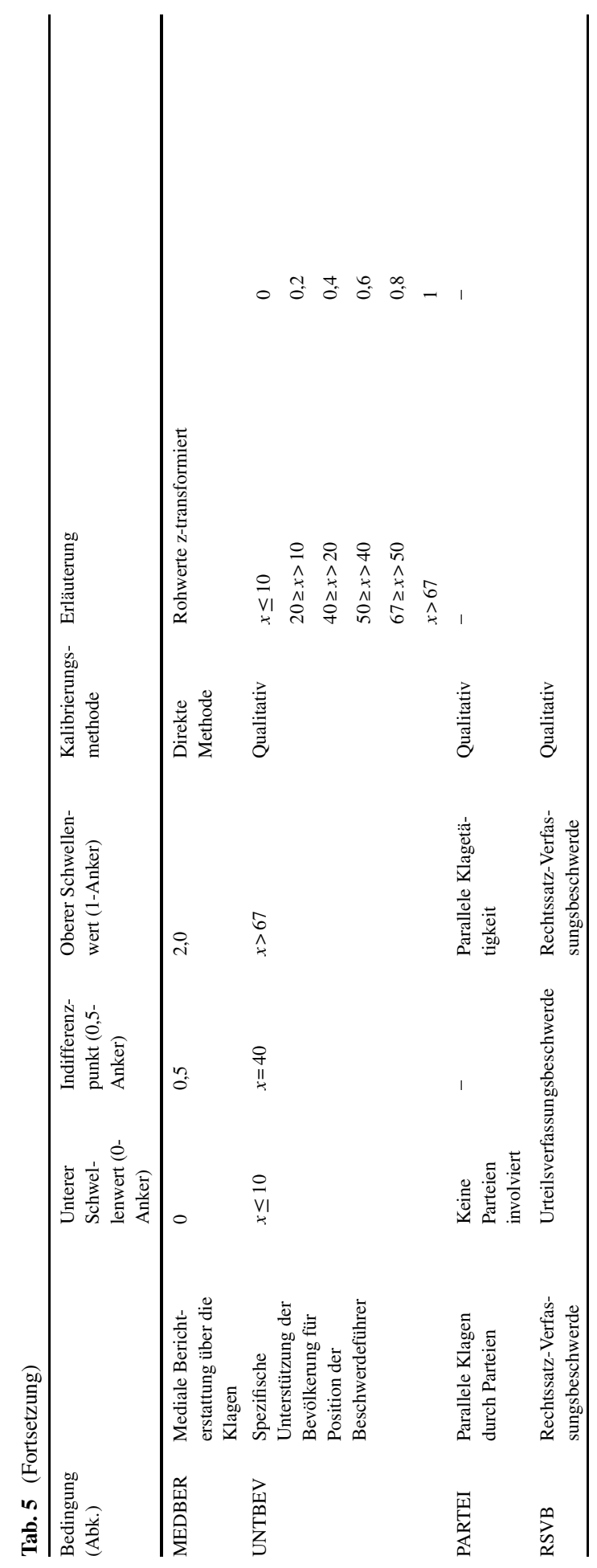




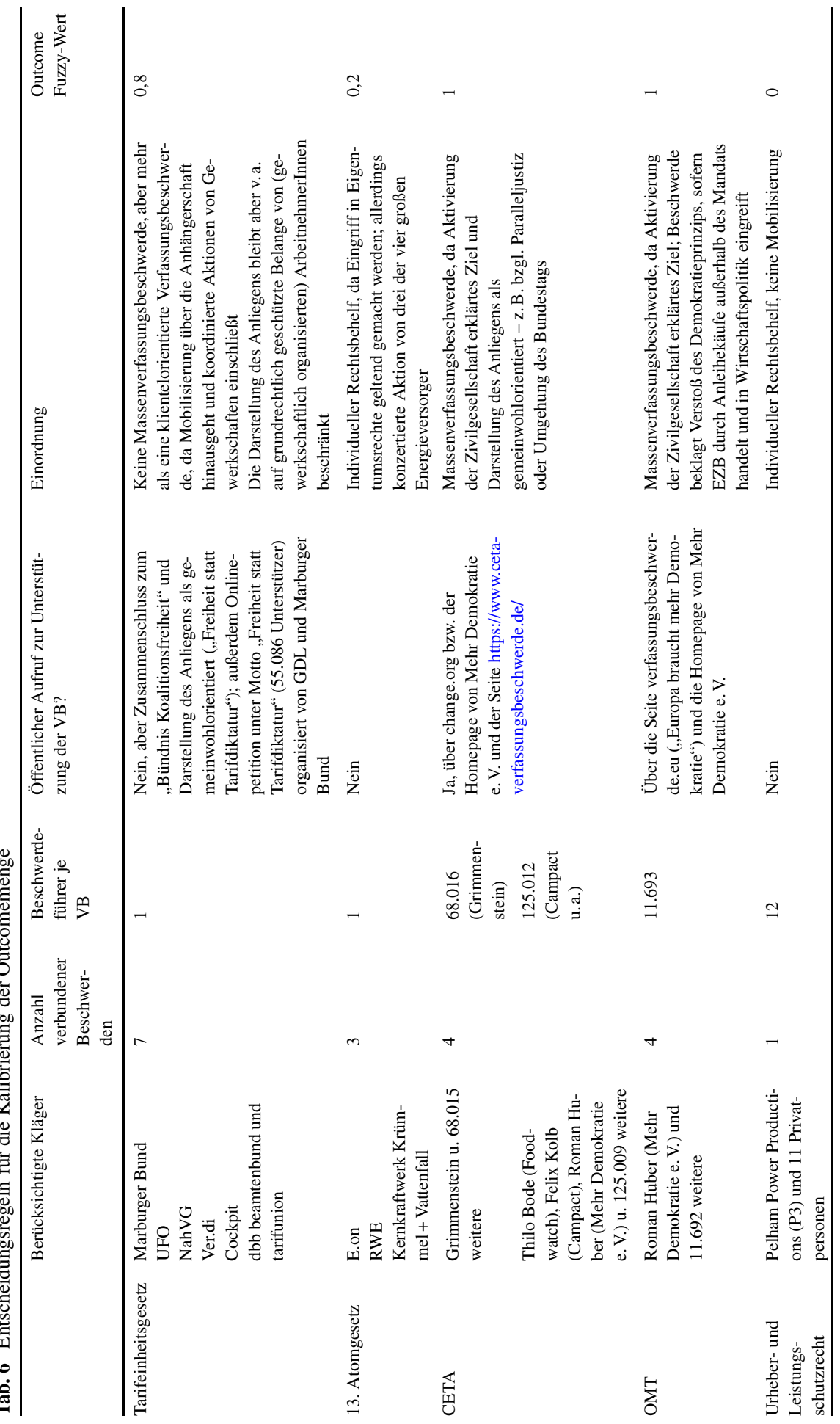




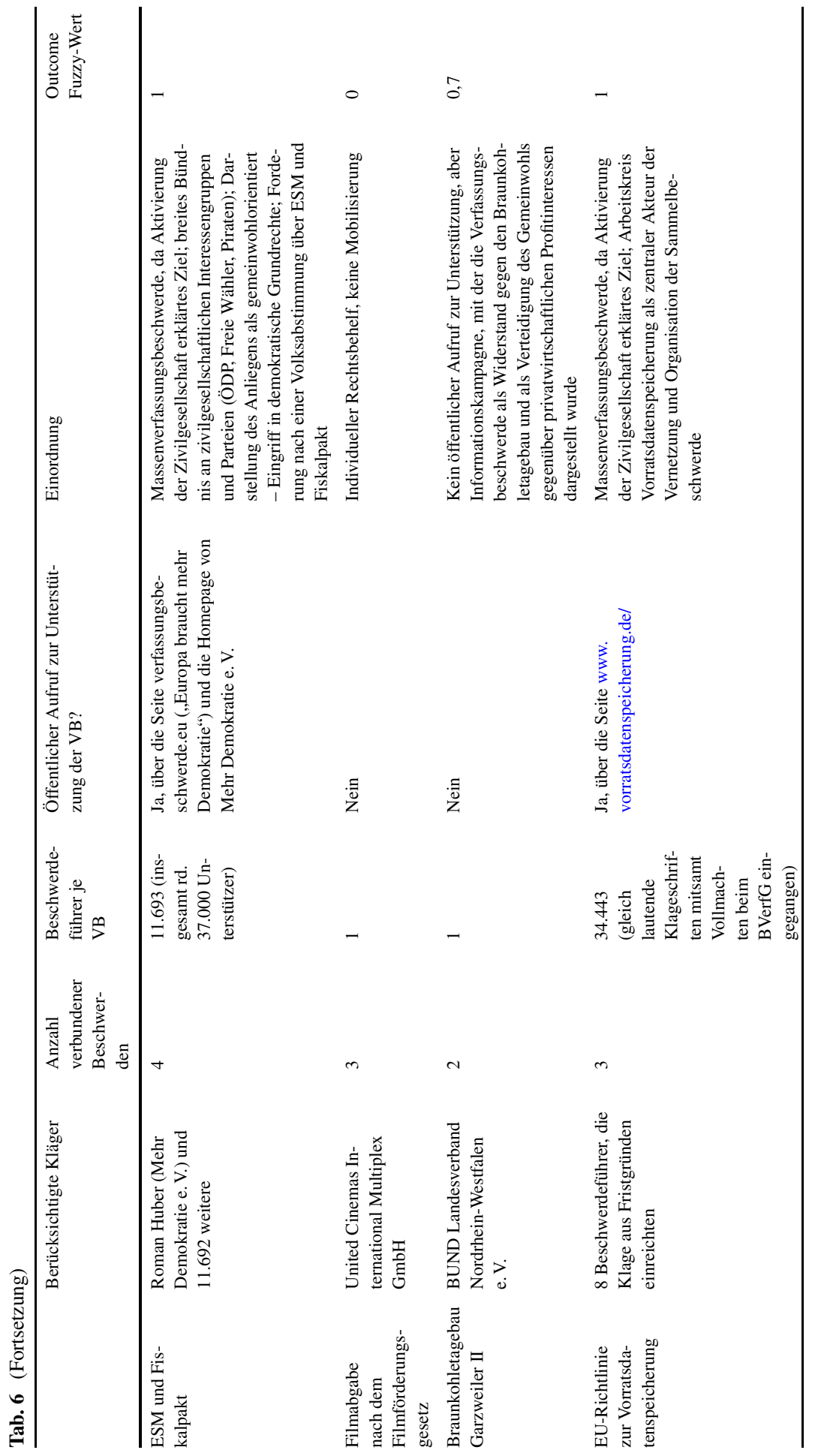




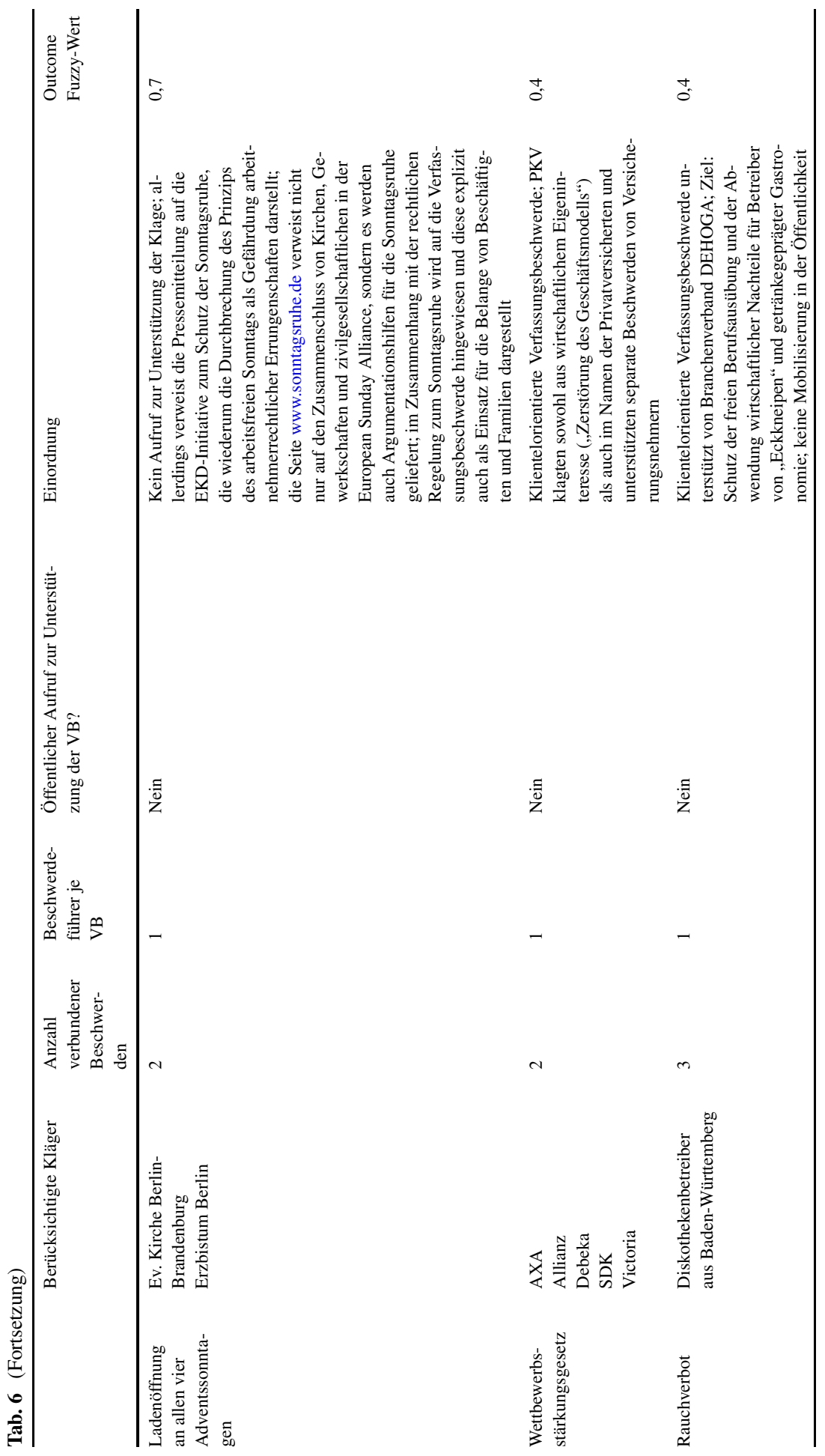




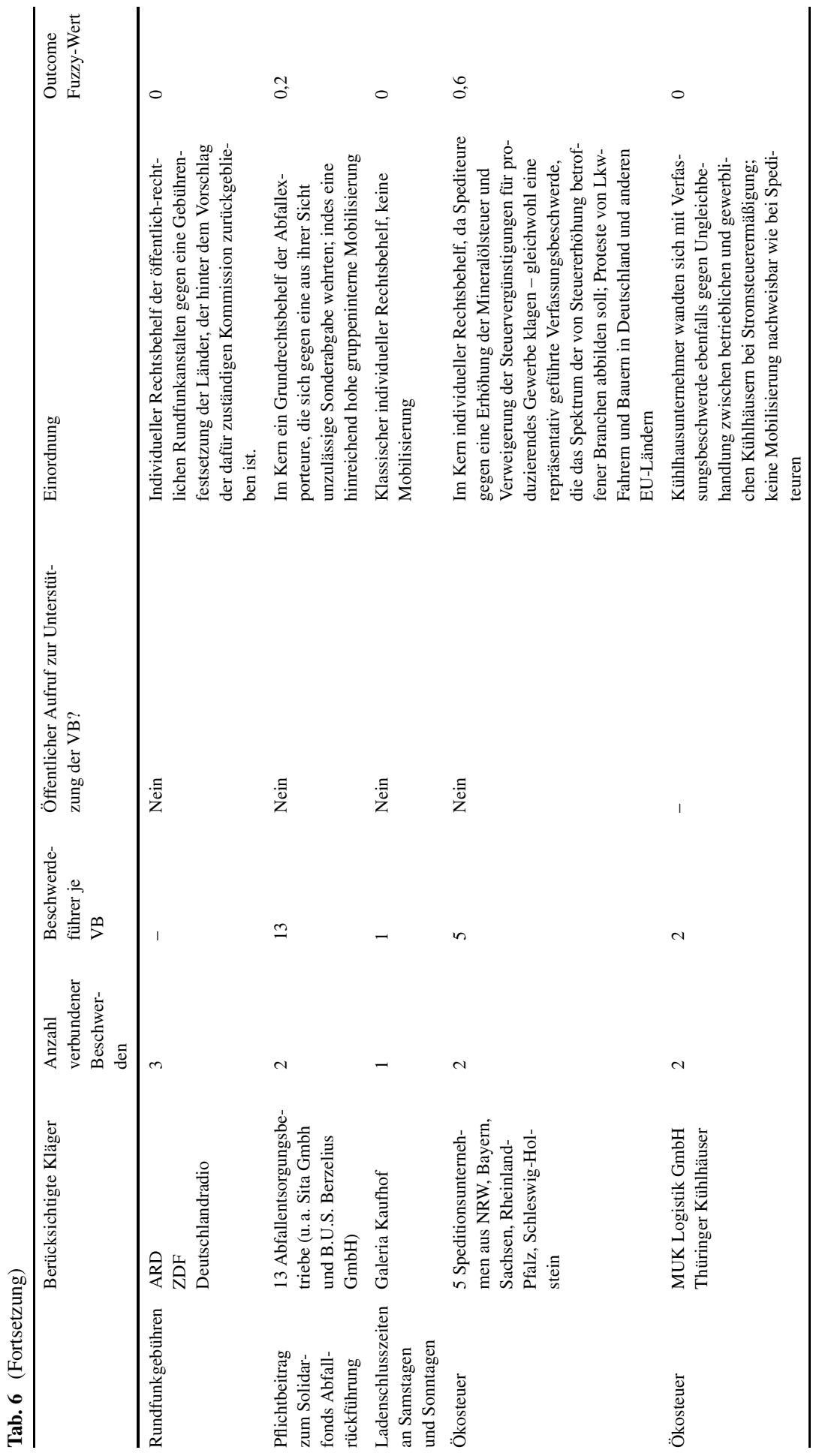




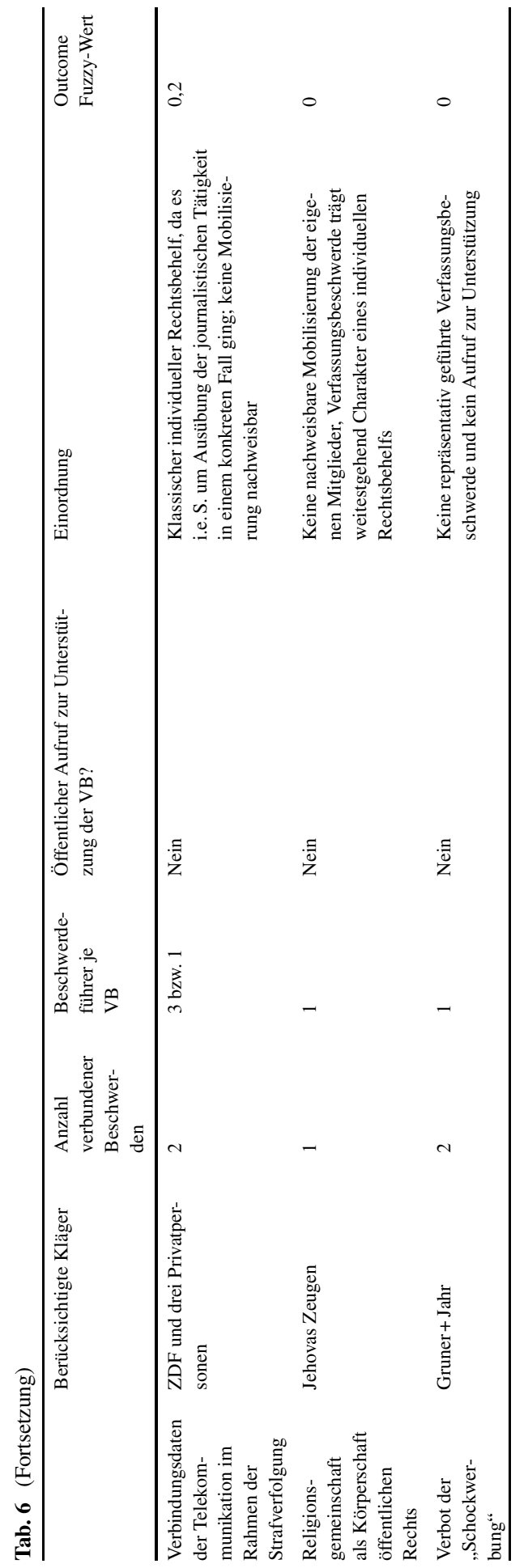


Tab. 7 Suchbegriffe für die Medienanalyse in der Datenbank Nexis zur Ermittlung der Medienberichterstattung

\begin{tabular}{ll}
\hline Fallgruppe & Suchbegriffe \\
\hline 1 & Tarifeinheit UND Verfassungsbeschwerde \\
2 & Atomgesetz ODER Atomausstieg UND Verfassungsbeschwerde \\
3 & CETA UND Verfassungsbeschwerde \\
4 & OMT UND EZB UND Verfassungsbeschwerde \\
5 & Sample UND Urheberrecht UND Verfassungsbeschwerde \\
6 & ESM ODER Fiskalpakt UND Verfassungsbeschwerde \\
7 & Filmabgabe ODER Filmförderungsgesetz UND Verfassungsbeschwerde \\
8 & Braunkohle ODER Tagebau ODER Garzweiler UND Verfassungsbeschwerde \\
9 & Vorratsdatenspeicherung UND Verfassungsbeschwerde \\
10 & Kirche UND verkaufsoffen UND Sonntag UND Berlin UND Verfassungsbeschwerde \\
11 & Private Krankenversicherung UND Wettbewerbsstärkungsgesetz UND Verfassungsbe- \\
12 & schwerde \\
13 & Rauchverbot UND Baden-Württemberg UND Verfassungsbeschwerde \\
14 & Rundfunkgebühren UND KEF UND Verfassungsbeschwerde \\
15 & Solidarfonds UND Abfall UND Verfassungsbeschwerde \\
16 & Ladenschluss UND Kaufhof UND Verfassungsbeschwerde \\
17 & Ökosteuer UND Spedition UND Kühlhäuser UND Verfassungsbeschwerde \\
19 & Strafverfolgung UND Telefon UND richterliche Anordnung UND Journalist UND \\
\hline
\end{tabular}




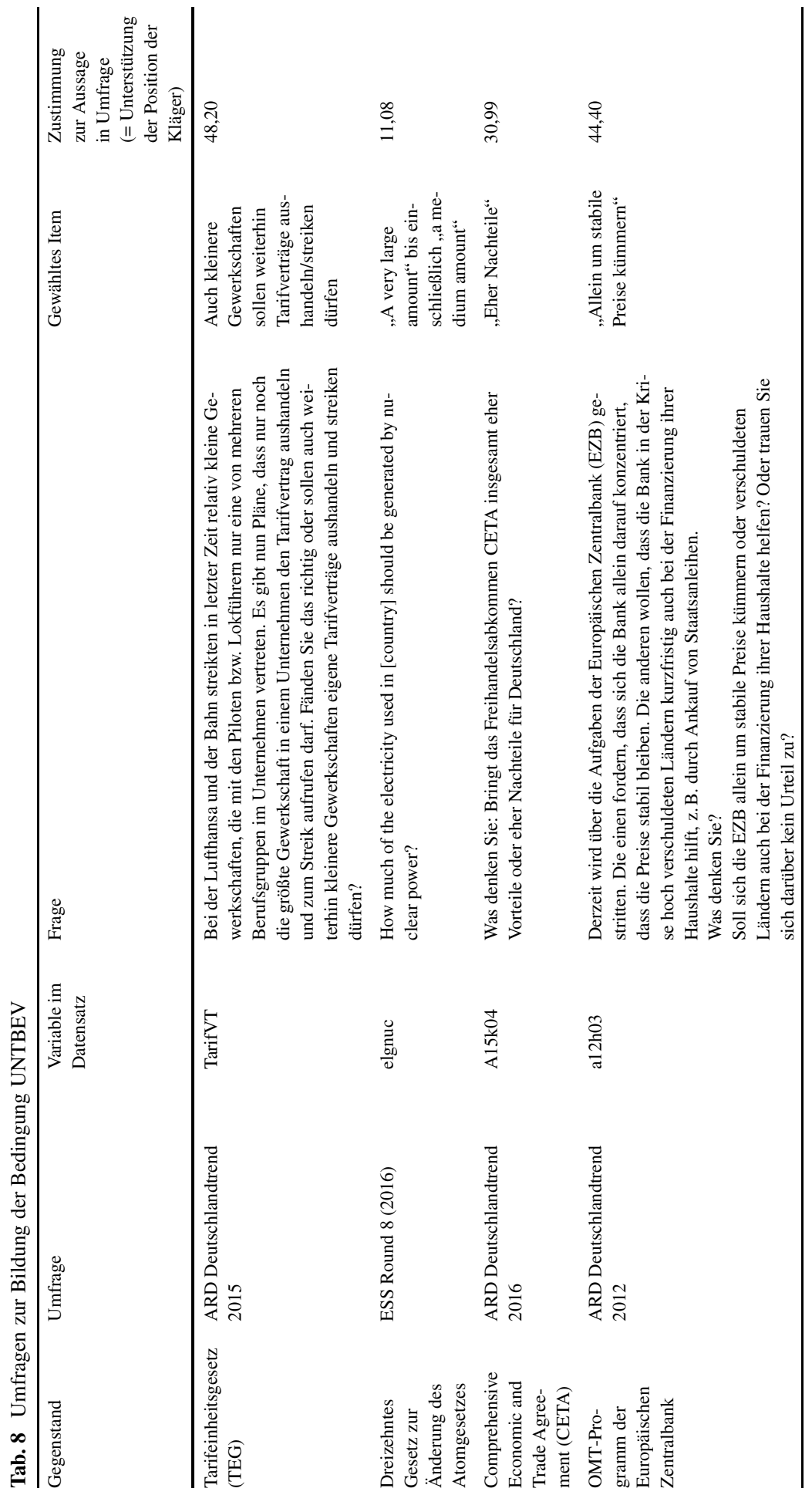




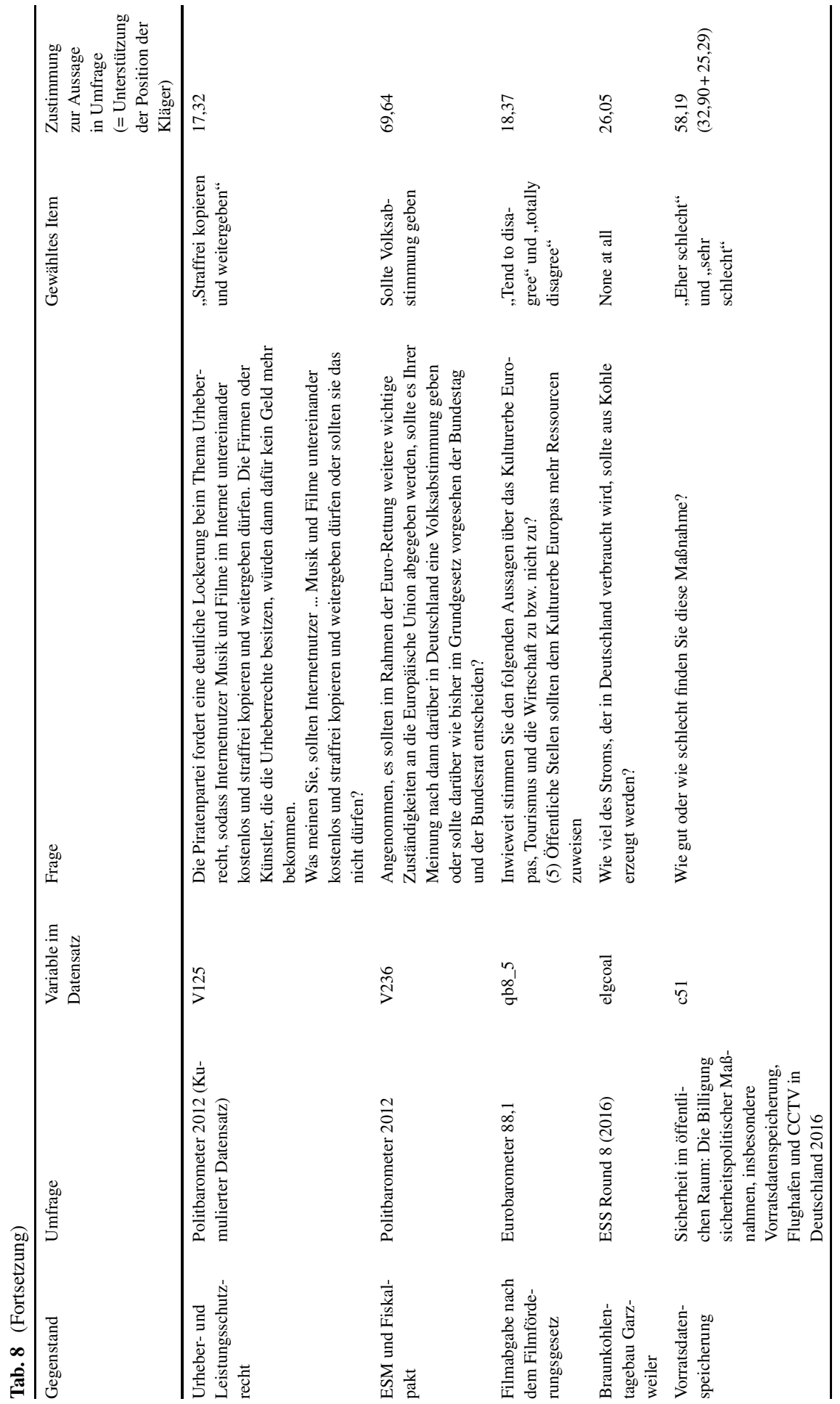




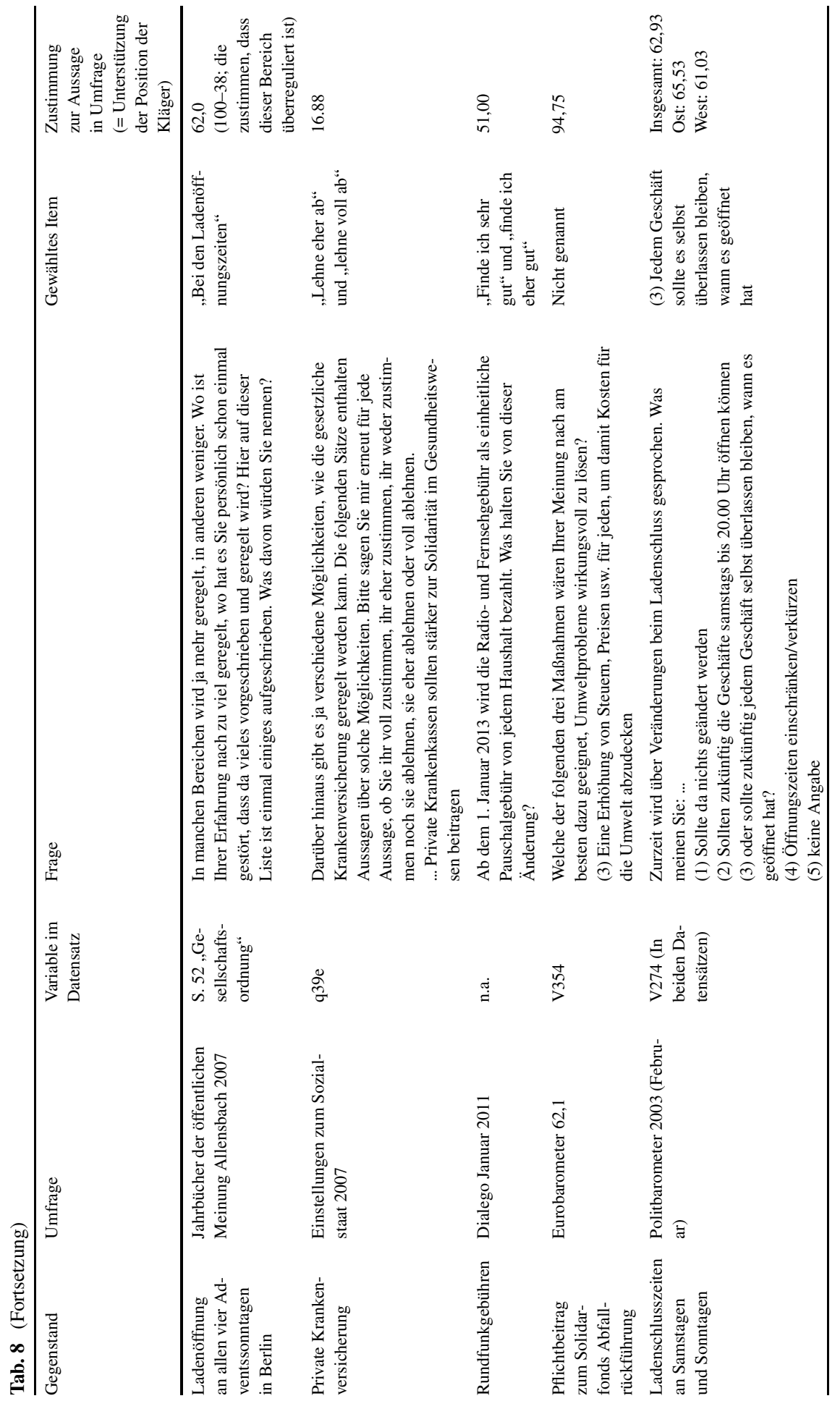




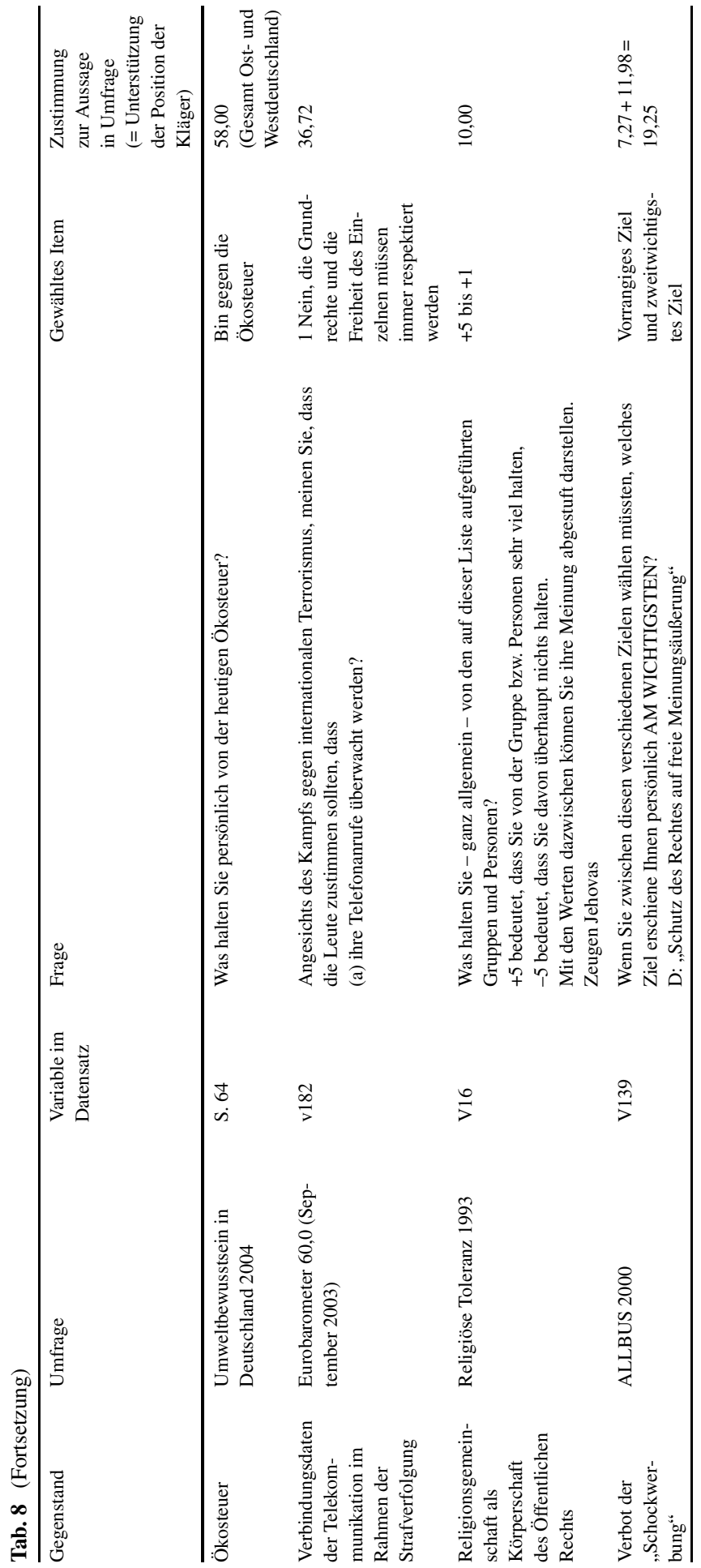


Tab. 9 Notwendige Bedingungen für das Outcome MOBIL

\begin{tabular}{lll}
\hline Bedingung & Konsistenz & Abdeckung \\
\hline INDMITGL & 0,697 & 0,791 \\
indmitgl & 0,303 & 0,213 \\
RESS & 0,250 & 0,550 \\
Ress & 0,877 & 0,474 \\
MEDBER & 0,485 & 0,645 \\
Medber & 0,742 & 0,478 \\
UNTBEV & 0,709 & 0,603 \\
Untbev & 0,527 & 0,470 \\
PARTEI & 0,329 & 1,000 \\
Partei & 0,671 & 0,340 \\
RSVB & $0,954^{\mathrm{a}}$ & 0,518 \\
Rsvb & 0,046 & 0,100 \\
\hline
\end{tabular}

${ }^{a}$ Konfigurationen liegen oberhalb des Schwellenwertes von 0,90 als notwendige Bedingung für das Outcome

Tab. 10 Lösungsgleichung für das Outcome MOBILI Komplexe Lösung

\begin{tabular}{lllll}
\hline Lösungspfad & $\begin{array}{l}\text { Rohab- } \\
\text { deckung }\end{array}$ & $\begin{array}{l}\text { Alleinige } \\
\text { Abdeckung }\end{array}$ & $\begin{array}{l}\text { Konsis- } \\
\text { tenz }\end{array}$ & $\begin{array}{l}\text { Exemplarische empirische } \\
\text { Fälle }\end{array}$ \\
\hline $\begin{array}{l}\text { INDMITGL*medber* } \\
\text { UNTBEV*partei* }\end{array}$ & 0,283 & 0,283 & 0,977 & $\begin{array}{l}\text { Gewerkschaften gegen Tarif- } \\
\text { einheitsgesetz }\end{array}$ \\
$\begin{array}{l}\text { RSVB } \\
\text { INDMITGL*ress*medber* }\end{array}$ & 0,122 & 0,025 & 1,000 & $\begin{array}{l}\text { Mehr Demokratie gegen } \\
\text { OMT; Grimmenstein via } \\
\text { PARTEI*RSVB }\end{array}$ \\
& & & $\begin{array}{l}\text { Change.org und Campact/ } \\
\text { foodwatch/Mehr Demokratie } \\
\text { gegen CETA }\end{array}$ \\
& 0,178 & 0,081 & 1,000 & $\begin{array}{l}\text { Mehr Demokratie gegen ESM } \\
\text { und Fiskalpakt; AK Vorrat ge- } \\
\text { gen Vorratsdatenspeicherung }\end{array}$ \\
$\begin{array}{l}\text { INDMITGL*ress* } \\
\text { UNTBEV*PARTEI* }\end{array}$ & & & & \\
RSVB & & & &
\end{tabular}

Konsistenz der Lösungsgleichung: 0,987

Abdeckung der Lösungsgleichung: 0,486

Tab. 11 Lösungsgleichung für das Outcome MOBILI Sparsamste Lösung

\begin{tabular}{llll}
\hline Lösungspfad & Rohabdeckung & Alleinige Abdeckung & Konsistenz \\
\hline INDMITGL*RSVB & 0,651 & 0,651 & 0,868 \\
\hline
\end{tabular}

Konsistenz der Lösungsgleichung: 0,868

Abdeckung der Lösungsgleichung: 0,651 
Tab. 12 Notwendige Bedingung für das Non-Outcome

\begin{tabular}{lll}
\hline Bedingung & Konsistenz & Abdeckung \\
\hline INDMITGL & 0,141 & 0,209 \\
indmitgl & 0,859 & 0,787 \\
RESS & 0,254 & 0,729 \\
ress & 0,843 & 0,594 \\
MEDBER & 0,379 & 0,657 \\
medber & 0,795 & 0,668 \\
UNTBEV & 0,556 & 0,611 \\
untbev & 0,641 & 0,747 \\
PARTEI & 0,000 & 0,000 \\
partei & $1,000^{\mathrm{a}}$ & 0,667 \\
RSVB & 0,688 & 0,482 \\
rsvb & 0,318 & 0,900 \\
\hline
\end{tabular}

${ }^{a}$ Konfigurationen liegen oberhalb des Schwellenwertes von 0,90 als notwendige Bedingung für das NonOutcome

Tab. 13 Wahrheitstafel für das Non-Outcome

\begin{tabular}{lllllllll}
\hline & \multicolumn{3}{l}{ Bedingungen } & & \multicolumn{5}{l}{ Outcome } \\
\hline INDMITGL & RESS & MEDBER & UNTBEV & PARTEI & RSVB & Mobil & Fallzahl & Konsistenz \\
0 & 0 & 0 & 1 & 0 & 1 & 1 & 7 & $0,931^{\mathrm{a}}$ \\
0 & 0 & 0 & 0 & 0 & 0 & 1 & 4 & $1,000^{\mathrm{a}}$ \\
0 & 0 & 0 & 0 & 0 & 1 & 1 & 4 & $0,885^{\mathrm{a}}$ \\
0 & 1 & 1 & 0 & 0 & 1 & 1 & 3 & $1,000^{\mathrm{a}}$ \\
0 & 0 & 0 & 1 & 0 & 0 & 1 & 1 & $1,000^{\mathrm{a}}$ \\
0 & 1 & 0 & 0 & 0 & 1 & 1 & 1 & $0,894^{\mathrm{a}}$ \\
0 & 1 & 0 & 1 & 0 & 1 & 1 & 1 & $1,000^{\mathrm{a}}$ \\
1 & 0 & 0 & 1 & 0 & 1 & 0 & 6 & 0,370 \\
1 & 0 & 0 & 0 & 0 & 0 & 0 & 2 & 0,794 \\
1 & 0 & 0 & 0 & 1 & 1 & 0 & 2 & 0,000 \\
1 & 0 & 1 & 1 & 1 & 1 & 0 & 2 & 0,000 \\
1 & 1 & 0 & 1 & 0 & 1 & 0 & 1 & 0,423 \\
1 & 0 & 0 & 1 & 1 & 1 & 0 & 1 & 0,000 \\
\hline
\end{tabular}

aKonfigurationen liegen oberhalb des Schwellenwertes von 0,80 als hinreichende Bedingung für das NonOutcome

Aus Platzgründen werden die logischen Rudimente nicht angeben

\section{Robustness checks}

QCA-spezifische Robustness Checks

In Anknüpfung an Schneider und Wagemann (2013, S. 285) kann von robusten Ergebnissen in QCA-Anwendungen dann gesprochen werden, wenn 
Tab. 14 Lösungsgleichung für das Outcome MOBILl Mittlere Lösung

\begin{tabular}{llll}
\hline Lösungspfad & $\begin{array}{l}\text { Rohab- } \\
\text { deckung }\end{array}$ & $\begin{array}{l}\text { Alleinige } \\
\text { Abdeckung }\end{array}$ & Konsistenz \\
\hline INDMITGL*ress*UNTBEV*partei*RSVB & 0,260 & 0,156 & 0,975 \\
INDMITGL*ress*untbev*PARTEI*RSVB & 0,118 & 0,025 & 1,000 \\
INDMITGL*ress*MEDBER*UNTBEV*RSVB & 0,276 & 0,000 & 1,000 \\
INDMITGL*ress*MEDBER*PARTEI*RSVB & 0,166 & 0,000 & 1,000 \\
\hline
\end{tabular}

Konsistenz der Lösungsgleichung: 0,986

Abdeckung der Lösungsgleichung: 0,451

- Sich über verschiedene Modellspezifikationen hinweg Konsistenz und Abdeckung der Lösungsgleichung nicht gravierend verändern

- Und wenn die Lösungsterme Unter-/Übermengenbeziehungen zueinander darstellen.

Zur Überprüfung der Robustheit der Ergebnisse wurde der Frequenzschwellenwert auf 2 heraufgesetzt. Dies schließt zwei Fälle aus der Analyse aus: Ver.di gegen das TEG und Mehr Demokratie gegen das OMT-Programm der EZB. Die Lösungsgleichung (mittlere Lösung) ist in Tab. 14 dargestellt.

Die Konsistenz und Abdeckung der Lösungsgleichung ändern sich nicht dramatisch, wenngleich der Lösungsterm komplexer ist.

Im Folgenden werden grafisch Untermengen-Übermengen-Beziehungen der Pfade aus Tab. 14 zu den Konfigurationen der mittleren Lösung aus Tab. 3 dargestellt. Jede Konfiguration stellt zu einer der „Hauptkonfigurationen“ eine vollständige Untermenge dar.

Untermengenbeziehung INDMITGL*UNTBEV*RSVB (y-Achse) und INDMITGL* ress*UNTBEV*partei*RSVB

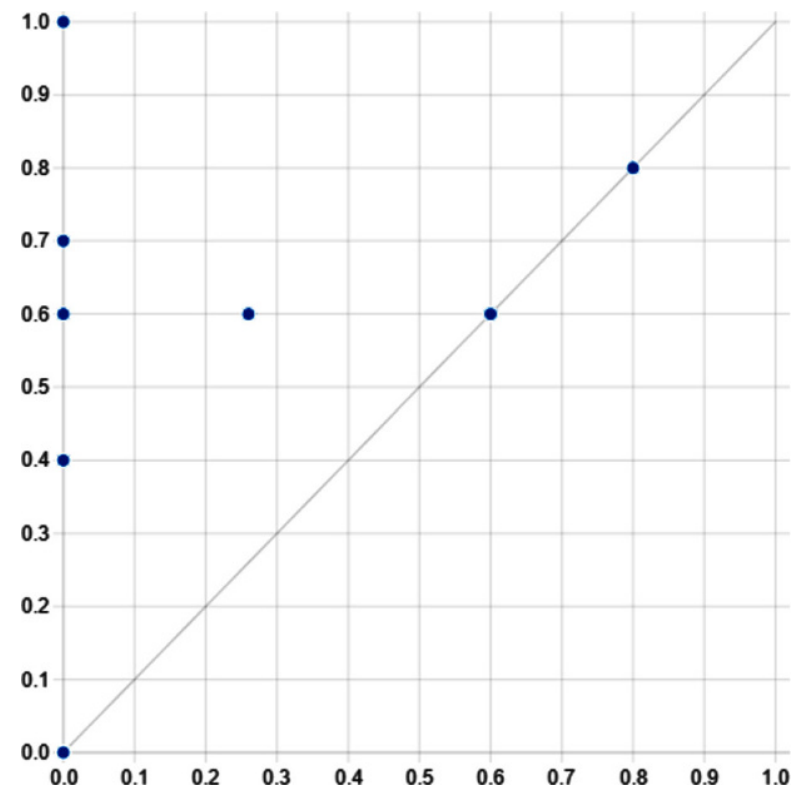


Untermengenbeziehung INDMITGL*ress*PARTEI*RSVB (y-Achse) und INDMITGL* ress*untbev*PARTEI*RSVB ( $x$-Achse)

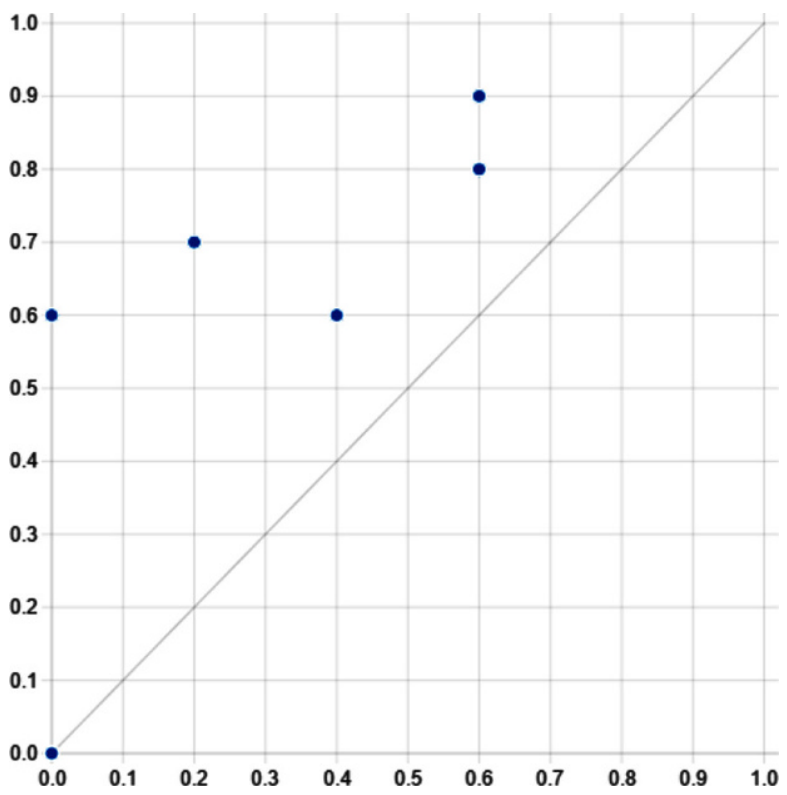

Analyse mit anderer Operationalisierung der Bedingung MEDBER Statt alle Einträge in Nexis vom Datum der Einreichung der Beschwerde bis einen Tag vor der Urteilsverkündung wurde für die folgende Analyse die Einträge in Nexis drei Monate vor der Einreichung der Verfassungsbeschwerde herangezogen. Damit lässt sich

Untermengenbeziehung INDMITGL*UNTBEV*RSVB (y-Achse) und INDMITGL* ress*MEDBER*UNTBEV* RSVB ( $x$-Achse)

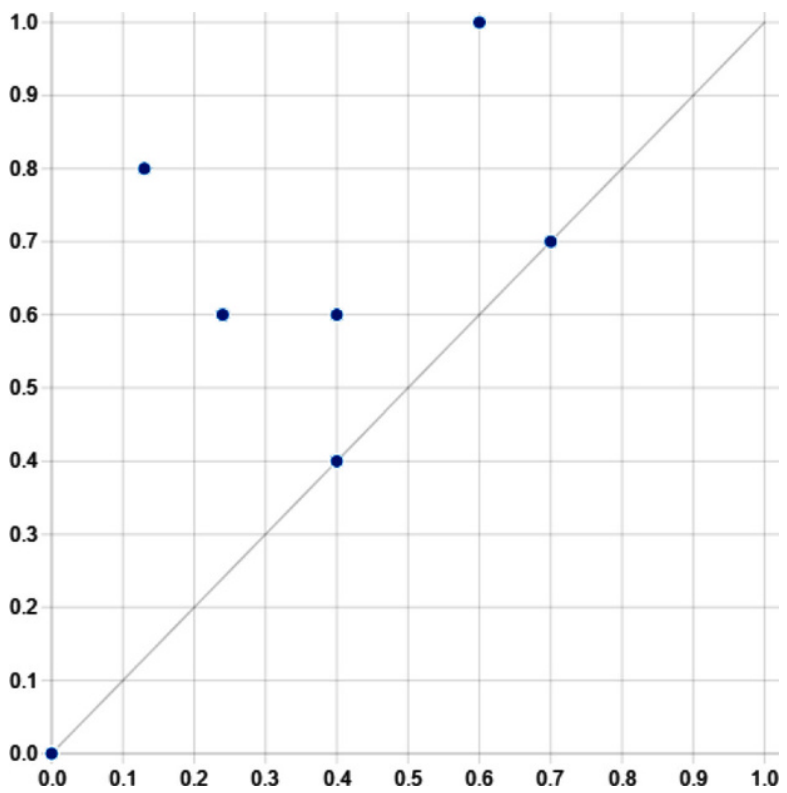


Untermengenbeziehung INDMITGL*ress*PARTEI*RSVB (y-Achse) und INDMITGL* ress*MEDBER*PARTEI* RSVB (x-Achse)

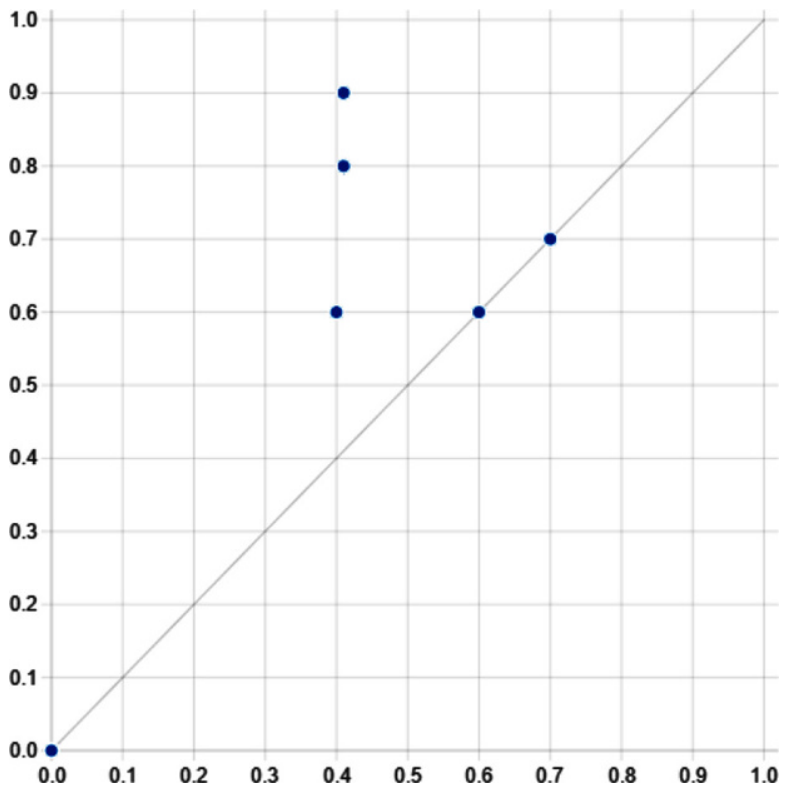

die erwartete mediale Aufmerksamkeit u. U. besser abbilden und es wird dem Umstand Rechnung getragen, dass Medien erst nach der Einreichung der Beschwerde umfassend berichten. Freilich ist auch mit der hier verwendeten Operationalisierung nicht ausgeschlossen, dass bereits die Ankündigung einer Verfassungsbeschwerde mediales Interesse und Nachrichtenwert erzeugt. Wie aus Tab. 15 hervorgeht, verändern sich Konsistenz und Abdeckung nur marginal. Es kommt indes ein dritter Pfad zur Lösungsgleichung hinzu.

Analyse mit anderer Operationalisierung der Bedingung RESS Da die Angaben zu den ausgestellten Hausausweisen zum Deutschen Bundestag aus zwei verschiedenen Jahren stammen und von zwei verschiedenen Stellen ausgestellt wurden

Tab. 15 Lösungsgleichung für das Outcome MOBILl Mittlere Lösung (andere Berechnung von MEDBER)

\begin{tabular}{lllll}
\hline Lösungspfad & $\begin{array}{l}\text { Rohab- } \\
\text { deckung }\end{array}$ & $\begin{array}{l}\text { Alleinige } \\
\text { Abdeckung }\end{array}$ & $\begin{array}{l}\text { Konsis- } \\
\text { tenz }\end{array}$ & $\begin{array}{l}\text { Exemplarische empirische } \\
\text { Fälle }\end{array}$ \\
\hline $\begin{array}{l}\text { INDMITGL*ress* } \\
\text { UNTBEV*RSVB }\end{array}$ & 0,438 & 0,080 & 0,985 & $\begin{array}{l}\text { AK Vorrat gegen Vorratsda- } \\
\text { tenspeicherung } \\
\text { Mehr Demokratie gegen ESM } \\
\text { INDMITGL*MEDBER* }\end{array}$ \\
$\begin{array}{l}\text { UNTBEV*RSVB } \\
\text { und Fiskalpakt; Gewerkschaf- } \\
\text { ten gegen Tarifeinheitsgesetz }\end{array}$ \\
$\begin{array}{l}\text { INDMITGL*ress* } \\
\begin{array}{l}\text { MEDBER*PARTEI* } \\
\text { RSVB }\end{array}\end{array}$ & 0,404 & 0,046 & 1,000 & $\begin{array}{l}\text { Grimmenstein via Change.org } \\
\text { und Campact/foodwatch/Mehr } \\
\text { Demokratie gegen CETA }\end{array}$ \\
\hline
\end{tabular}

Konsistenz der Lösungsgleichung: 0,988

Abdeckung der Lösungsgleichung: 0,543 
Tab. 16 Lösungsgleichung für das Outcome MOBILI Mittlere Lösung (andere Berechnung von RESS)

\begin{tabular}{lllll}
\hline Lösungspfad & $\begin{array}{l}\text { Rohab- } \\
\text { deckung }\end{array}$ & $\begin{array}{l}\text { Alleinige } \\
\text { Abdeckung }\end{array}$ & $\begin{array}{l}\text { Konsis- } \\
\text { tenz }\end{array}$ & $\begin{array}{l}\text { Exemplarische empirische } \\
\text { Fälle }\end{array}$ \\
\hline $\begin{array}{l}\text { INDMITGL*ress* } \\
\text { UNTBEV*RSVB }\end{array}$ & 0,450 & 0,278 & 1,000 & $\begin{array}{l}\text { Mehr Demokratie gegen ESM } \\
\text { und Fiskalpakt; Gewerkschaf- } \\
\text { ten gegen Tarifeinheitsgesetz } \\
\text { Grimmenstein via Change.org }\end{array}$ \\
$\begin{array}{l}\text { INDMITGL*ress* } \\
\text { PARTEI*RSVB }\end{array}$ & 0,237 & 0,059 & 1,000 & $\begin{array}{l}\text { Gnd Campact/foodwatch/Mehr } \\
\text { Demokratie gegen CETA; AK } \\
\text { Vorrat gegen Vorratsdaten- } \\
\text { speicherung; Mehr Demokra- } \\
\text { tie gegen OMT-Programm }\end{array}$ \\
\hline
\end{tabular}

Konsistenz der Lösungsgleichung: 1,000

Abdeckung der Lösungsgleichung: 0,496

(die Zahlen aus dem Jahr 2015 beziehen sich auf die Hausausweise, die über die Fraktionen ausgestellt wurden, während die Zahlen aus dem Jahr 2017 auf Hausausweise bezieht, die von der Bundestagsverwaltung ausgestellt wurden), wurde die Fuzzy-set-QCA mit einem Index für Ressourcen durchgeführt, der nur drei Größen einbezieht: Lobbyisten in VZÄ in Brüssel, Lobbyingbudget (auf EU-Ebene) und Größe der Belegschaft. Tab. 16 verdeutlicht, dass sich die Ergebnisse nicht substanziell verändern. Sie entsprechen vielmehr weitgehend den zwei Pfaden, die sich auch im Zusammenhang mit einem höheren Frequenz-Schwellenwert ergaben. Indes ist die Gesamtabdeckung der Lösungsgleichung etwas geringer, die Konsistenz dafür etwas höher. 


\section{Statistische Robustness Checks}

Tab. 17 OLS-Regression (standardisierte Rohwerte links und Fuzzy-Werte rechts)

\begin{tabular}{llllll}
\hline & MOBIL & & & MOBIL & \\
\hline Individuelle Mitglied- & $0,496^{* * *}$ & $0,331^{*}$ & INDMITGL & $0,489^{* * *}$ & $0,314^{*}$ \\
schaft & $(0,068)$ & $(0,140)$ & & $(0,068)$ & $(0,136)$ \\
Ressourcen & $-0,001$ & 0,002 & RESS & $-0,078$ & $-0,064$ \\
& $(0,144)$ & $(0,142)$ & & $(0,260)$ & $(0,255)$ \\
Medienberichterstattung & $-0,013$ & $-0,013$ & MEDBER & $-0,018$ & $-0,020$ \\
& $(0,041)$ & $(0,040)$ & & $(0,150)$ & $(0,147)$ \\
Öffentliche Meinung & $-0,026$ & $-0,034$ & UNTBEV & $-0,117$ & $-0,143$ \\
positiv & $(0,033)$ & $(0,033)$ & & $(0,115)$ & $(0,114)$ \\
Parteien klagen parallel & $0,261^{*}$ & $0,227^{\dagger}$ & PARTEI & $0,315^{* *}$ & $0,259^{*}$ \\
& $(0,110)$ & $(0,111)$ & & $(0,113)$ & $(0,117)$ \\
Rechtssatz & $0,327^{* * *}$ & $0,269^{* *}$ & RSVB & $0,342^{* * *}$ & $0,279^{* *}$ \\
Individuelle Mitglied- & $(0,082)$ & $(0,091)$ & & $(0,084)$ & $(0,092)$ \\
Schaft*Rechtssatz & - & 0,218 & INDMITGL* & - & 0,231 \\
Konstante & & $(0,161)$ & RSVB & & $(0,157)$ \\
& $-0,063$ & $-0,021$ & Konstante & 0,035 & 0,086 \\
$N$ & $(0,075)$ & $(0,081)$ & & $(0,135)$ & $(0,137)$ \\
Korr.R & & & & & 35 \\
\hline
\end{tabular}

Abhängige Variable: Grad politischer Mobilisierung (Fuzzy-kalibrierte Outcomemenge) Standardfehler in Klammern ${ }^{\dagger} p<0,10{ }^{*} p<0,05 ;{ }^{* *} p<0,01 ;{ }^{* * *} p<0,001$

Tab. 18 Ordinale logistische Regression (standardisierte Rohwerte links und Fuzzy-Werte rechts)

\begin{tabular}{llllll}
\hline & MOBIL & & \multicolumn{3}{l}{ MOBIL } \\
\hline Individuelle Mitglied- & 21,046 & 22,590 & INDMITGL & 21,697 & 23,036 \\
schaft & $(39,323)$ & $(20,359)$ & & $(19,964)$ & $(21,311)$ \\
Ressourcen & 0,385 & 0,385 & RESS & 0,557 & 0,557 \\
& $(1,782)$ & $(2,280)$ & & $(2,849)$ & $(4,371)$ \\
Medienberichterstattung & $-0,396$ & $-0,396$ & MEDBER & $-1,846$ & $-1,846$ \\
& $(37,587)$ & $(3,179)$ & & $(2,050)$ & $(3,406)$ \\
Öffentliche Meinung & $-0,550$ & $-0,550$ & UNTBEV & $-2,891$ & $-2,891$ \\
positiv & $(4,939)$ & $(0,869)$ & & $(3,169)$ & $(3,024)$ \\
Parteien klagen parallel & $43,359^{*}$ & $43,360^{* * *}$ & PARTEI & $50,067^{* * *}$ & $50,422^{* * *}$ \\
& $(17,984)$ & $(7,279)$ & & $(9,543)$ & $(10,112)$ \\
Rechtssatz & 20,686 & $22,230^{* * *}$ & RSVB & 21,755 & $23,094^{* * *}$ \\
Individuelle Mitglied- & $(28,150)$ & $(3,475)$ & & $(13,739)$ & $(3,047)$ \\
schaft*Rechtssatz & - & $-1,607$ & INDMITGL* & & $-1,392$ \\
$N$ & & $(9,795)$ & RSVB & & $(10,100)$ \\
Pseudo $R^{2}$ & 35 & 35 & - & 35 & 35 \\
\hline
\end{tabular}

Abhängige Variable: Grad politischer Mobilisierung (Fuzzy-kalibrierte Outcomemenge) Bootstrap-Standardfehler in Klammern ${ }^{*} p<0,05 ;{ }^{* *} p<0,01 ;{ }^{* * *} p<0,001$ 
Tab. 19 Deskriptive Statistik der Outcome- und Bedingungsmengen und der stetigen Variablen

\begin{tabular}{|c|c|c|c|c|c|}
\hline & & Mittelwert & $\begin{array}{l}\text { Standard- } \\
\text { abweichung }\end{array}$ & Minimum & Maximum \\
\hline MOBIL & 35 & 0,4342857 & 0,3733338 & 0 & 1 \\
\hline INDMITGL & 35 & 0,3828571 & 0,4792676 & 0 & 1 \\
\hline MEDBER & 35 & 0,326736 & 0,2784658 & 0,1301832 & 0,9789434 \\
\hline UNTBEV & 35 & 0,5142857 & 0,288141 & 0 & 1 \\
\hline PARTEI & 35 & 0,1428571 & 0,3550358 & 0 & 1 \\
\hline RSVB & 35 & 0,8 & 0,4058397 & 0 & 1 \\
\hline Ressourcen (normalisiert) & 35 & 0,1927311 & 0,2570186 & 0 & 1 \\
\hline $\begin{array}{l}\text { Medienberichterstattung ( } \mathrm{z}- \\
\text { transformiert) }\end{array}$ & 35 & $2,55 \mathrm{e}-09$ & 1 & $-0,7662268$ & 2,419631 \\
\hline $\begin{array}{l}\text { Öffentliche Meinung (z-transfor- } \\
\text { miert) }\end{array}$ & 35 & $3,19 \mathrm{e}-09$ & 1 & $-1,319739$ & 2,421207 \\
\hline$N$ & 35 & - & - & - & - \\
\hline
\end{tabular}

\section{Literatur}

Aden, Hartmut. 2014. Einflussnahme oder Entpolitisierung? Menschen- und Bürgerrechtsorganisationen als Akteure der Sicherheitskultur. In Dimensionen der Sicherheitskultur, Hrsg. Hans-Jürgen Lange, Michaela Wendekamm, und Christian Endreß, 235-248. Wiesbaden: Springer VS.

Andersen, Ellen Ann. 2005. Out of the closets and into the courts. Legal opportunity structure and gay rights litigation. Ann Arbor: University of Michigan Press.

Berkhout, Joost, Brendan J. Carroll, Caelesta Braun, Adam W. Chalmers, Tine Destrooper, David Lowery, Simon Otjes, und Anne Rasmussen. 2015. Interest organizations across economic sectors: explaining interest group density in the European Union. Journal of European Public Policy 22(4):462-480.

Beyers, Jan. 2008. Policy issues, organisational format and the political strategies of interest organisations. West European Politics 31(6):1188-1211.

Blankenburg, Erhard. 1995. Mobilisierung des Rechts. Eine Einführung in die Rechtssoziologie. Berlin, Heidelberg: Springer.

Blankenburg, Erhard. 1998. Die Verfassungsbeschwerde - Nebenbühne der Politik und Klagemauer von Bürgern. Kritische Justiz 31(2):203-218.

Bomberg, Elizabeth. 2012. Mind the (mobilization) gap: comparing climate activism in the United States and European Union. Review of Policy Research 29(3):408-430.

Bouwen, Pieter. 2002. Corporate lobbying in the European Union: the logic of access. Journal of European Public Policy 9(3):365-390.

Brandenburg, Jens. 2014. Lady justice's delay: judicial policy bargaining and the duration of senate proceedings at the German federal constitutional court. Diss. Mannheim: Univ. Mannheim. https:// madoc.bib.uni-mannheim.de/37556/.

Brunner, Georg. 2002. Der Zugang des Einzelnen zur Verfassungsgerichtsbarkeit im europäischen Raum. In Jahrbuch des öffentlichen Rechts der Gegenwart, Bd. 50, Hrsg. Peter Häberle, 191-256. Tübingen: J.C.B. Mohr.

de Bruycker, Iskander, und Jan Beyers. 2015. Balanced or biased? Interest groups and legislative lobbying in the European news media. Political Communication 32(3):453-474.

Burstein, Paul. 1991. Legal mobilization as a social movement tactic: the struggle for equal employment opportunity. American Journal of Sociology 96(5):1201-1225.

Cortner, Richard. 1968. Strategies and tactics of litigants in constitutional cases. Journal of Public Law 17(2):287-307.

Dannemann, Gerhard. 1994. Constitutional complaints: the European perspective. The International and Comparative Law Quarterly 43(1):142-153. 
Diani, Mario. 2013. Organizational fields and social movement dynamics. In The future of social movement research: dynamics, mechanisms, and processes, Hrsg. Jacquelien van Stekelenburg, Conny Roggeband, und Bert Klandermans, 145-168. Minneapolis: University of Minnesota Press.

Döring, Nicola, und Jürgen Bortz. 2016. Forschungsmethoden und Evaluation in den Sozial- und Humanwissenschaften, 5. Aufl., Berlin, Heidelberg: Springer.

Dür, Andreas, und Gemma Mateo. 2013. Gaining access or going public? Interest group strategies in five European countries. European Journal of Political Research 52(5):660-686.

Epp, Charles R. 2008a. Law as an Instrument of Social Reform. In The Oxford handbook of law and politics, Hrsg. Keith E. Whittington, 595-613. Oxford: Oxford University Press.

Epp, Charles R. 2008b. The rights revolution. Lawyers, activists, and supreme courts in comparative perspective, 4. Aufl., Chicago: University of Chicago Press.

Fuchs, Gesine. 2019. Rechtsmobilisierung: Rechte kennen, Rechte nutzen und Recht bekommen. In Interdisziplinäre Rechtsforschung: Eine Einführung in die geistes- und sozialwissenschaftliche Befassung mit dem Recht und seiner Praxis, Bd. 39, Hrsg. Christian Boulanger, Julika Rosenstock, und Tobias Singelnstein, 243-256. Wiesbaden: Springer VS.

Galanter, Marc. 1974. Why the haves come out ahead: speculations on the limits of legal change. Law Society Review 9(1):95-160.

Gawron, Thomas, und Rudolf Schäfer. 1976. Justiz und organisierte Interessen in der BRD. In Legitimationsprobleme Politischer Systeme: Tagung der Deutschen Vereinigung für Politische Wissenschaft. Duisburg, 1975. Politische Vierteljahresschrift Sonderheft, 7/1976., Hrsg. Peter Kielmansegg, 217-269. Opladen: Westdeutscher Verlag.

Grant, Wyn. 2000. Pressure groups and British politics. Contemporary political studies. Houndmills, Basingstoke, Hampshire, New York: Macmillan Press.

Graser, Alexander, und Christian Helmrich (Hrsg.). 2019. Strategic Litigation. Begriff und Praxis. BadenBaden: Nomos.

Gusy, Christoph. 2015. Die Verfassungsbeschwerde. In Handbuch Bundesverfassungsgericht im politischen System, 2. Aufl., Hrsg. Robert Christoph van Ooyen, Martin H.W. Möllers, 333-347. Wiesbaden: Springer VS.

Hahn, Lisa. 2019. Strategische Prozessführung. Zeitschrift für Rechtssoziologie 39(1):5-32.

Handler, Joel F. 1978. Social movements and the legal system. A theory of law reform and social change. New York: Academic Press.

Hilson, Chris. 2002. New social movements: the role of legal opportunity. Journal of European Public Policy 9(2):238-255.

Hönnige, Christoph. 2010. Beyond Judicialization: why we need more comparative research about constitutional courts. European Political Science 10(3):346-358.

Jordan, Grant, Darren Halpin, und William Maloney. 2004. Defining interests: disambiguation and the need for new distinctions. British Journal of Politics and International Relations 6(2):195-212.

Klüver, Heike. 2013. Lobbying in the European Union. Interest groups, lobbying coalitions, and policy change. Oxford: Oxford University Press.

Köhler, Andreas. 2012. Litigation-PR in Verfassungsfragen: Die Verfassungsklage als oppositionelles PRInstrument. In Litigation-PR: Alles was Recht ist, Hrsg. Lars Rademacher, Alexander Schmitt-Geiger, 203-217. Wiesbaden: VS.

Kollman, K. 1998. Outside lobbying: public opinion and interest group strategies. Princeton: Princeton University Press.

Koopmans, Ruud. 1999. Political. Opportunity. Structure. Some splitting to balance the lumping. Sociological Forum 14(1):93-105.

Krehbiel, Jay N. 2016. The politics of judicial procedures: the role of public oral hearings in the German constitutional court. American Journal of Political Science 60(4):990-1005.

Mahoney, Christine. 2007. Lobbying Success in the United States and the European Union. Journal of Public Policy 27(1):35-56.

McCann, Michael W. 2008. Litigation and legal mobilization. In The Oxford handbook of law and politics, Hrsg. Keith E. Whittington, 522-540. Oxford: Oxford University Press.

McCann, Michael W. 2016. Legal mobilization and social reform movements: notes on theory and its application. In Law and social movements, Hrsg. Michael W. McCann, 3-32. London, New York: Routledge.

McCarthy, John D., und Mayer N. Zald. 1977. Resource mobilization and social movements: a partial theory. The American Journal of Sociology 82(6):1212-1241. 
McCown, Margaret. 2009. Interest groups and the European court of justice. In Lobbying the European Union: institutions, actors, and issues, Hrsg. David Coen, Jeremy J. Richardson, 89-104. Oxford, New York: Oxford University Press.

Nocun, Katharina, und Patrick Breyer. 2014. Distributed Denial of Service: Dieses Grundrecht ist auf ihrem Gerät nicht verfügbar. In Gesellschaftliche Verantwortung in der digital vernetzten Welt, Hrsg. Peter Bittner, Stefan Hügel, Hans-Jörg Kreowski, Dietrich Meyer-Ebrecht, und Britta Schinzel, 37-44. Berlin, Münster: LIT.

Oberschall, Anthony R. 1973. Social conflict and social movements, 2. Aufl., Englewood Cliffs: PrenticeHall.

Olson, Mancur. 1971. The logic of collective action. Public goods and the theory of groups. Harvard economic studies. Princeton: Harvard University Press.

Pestalozza, Christian. 1991. Verfassungsprozeßrecht. Die Verfassungsgerichtsbarkeit des Bundes und der Länder. Mit einem Anhang zum internationalen Rechtsschutz. 3. Aufl., München: Beck.

Pralle, Sarah B. 2003. Venue shopping, political strategy, and policy change: the internationalization of Canadian forest advocacy. Journal of Public Policy 23(3):233-260.

Rucht, Dieter. 2001. Lobbying or protest? Strategies to influence EU environmental policies. In Contentious Europeans: protest and politics in an integrating Europe, Hrsg. Douglas R. Imig, Sidney G. Tarrow, 125-142. Lanham, Oxford: Rowman \& Littlefield.

Rucht, Dieter. 2008. The impact of national contexts on social movement structures: a cross-movement and cross-national comparison. In Comparative perspectives on social movements: political opportunities, mobilizing structures, and cultural framings, 9. Aufl., Hrsg. Doug McAdam, John D. McCarthy, und Mayer N. Zald, 185-204. Cambridge: Cambridge University Press.

Salisbury, Robert H. 1969. An exchange theory of interest groups. Midwest Journal of Political Science 13(1):1-32.

Schattschneider, Elmer E. 1975. The semisovereign people. A realist's view of democracy in America. Boston: Wadsworth.

Scheingold, Stuart A. 2004. The politics of rights. Lawyers, public policy, and political change, 2. Aufl., Ann Arbor: University of Michigan Press.

Schneider, Carsten Q., und Claudius Wagemann. 2009. Standards guter Praxis in Qualitative Comparative Analysis (QCA) und Fuzzy-Sets. In Methoden der vergleichenden Politik- und Sozialwissenschaft: Neue Entwicklungen und Anwendungen, Hrsg. Susanne Pickel, Detlef Jahn, Hans-Joachim Lauth, und Gert Pickel, 387-412. Wiesbaden: VS.

Schneider, Carsten Q., und Claudius Wagemann. 2013. Set-theoretic methods for the social sciences. A guide to qualitative comparative analysis. Strategies for social inquiry. Cambridge: Cambridge University Press.

Schreier, Christian. 2011. Die Massenverfassungsbeschwerde beim Bundesverfassungsgericht. Versuche der Revision von Rechtsnormen durch Bürgerinitiativen. Berlin: MAECENATA Institut für Philanthropie und Zivilgesellschaft an der Humboldt-Universität zu Berlin.

Sternberg, Sebastian, Thomas Gschwend, Caroline Wittig, und Benjamin G. Engst. 2015. Zum Einfluss der öffentlichen Meinung auf Entscheidungen des Bundesverfassungsgerichts: Eine Analyse von abstrakten Normenkontrollen sowie Bund-Länder-Streitigkeiten 1974-2010. Politische Vierteljahresschrift 56(4):570-598.

Stone Sweet, Alec. 2000. Governing with judges. Constitutional politics in Europe. Oxford, New York: Oxford University Press.

Thierse, Stefan, und Sanja Badanjak. 2020. Opposition in the EU multi-level polity. Legal mobilization against the data retention directive. Cham: Palgrave Macmillan.

Tresch, Anke, und Manuel Fischer. 2015. In search of political influence: Outside lobbying behaviour and media coverage of social movements, interest groups and political parties in six Western European countries. International Political Science Review 36(4):355-372.

de Visser, Maartje. 2015. Constitutional review in Europe. A comparative analysis. Oxford: Hart Publishing.

Zemans, Frances Kahn. 1983. Legal mobilization: the neglected role of the law in the political system. American Political Science Review 77(3):690-703.

Zuck, Rüdiger. 2013. Das Recht der Verfassungsbeschwerde, 4. Aufl., München: Beck. 